\title{
Fault Diagnosis for Satellite Sensors and Actuators using Nonlinear Geometric Approach and Adaptive Observers
}

Baldi, P.; Blanke, Mogens; Castaldi, P.; Mimmo, N.; Simani, S.

Published in:

International Journal of Robust and Nonlinear Control

Link to article, DOI:

$10.1002 /$ rnc.4083

Publication date:

2018

Document Version

Peer reviewed version

Link back to DTU Orbit

Citation (APA):

Baldi, P., Blanke, M., Castaldi, P., Mimmo, N., \& Simani, S. (2018). Fault Diagnosis for Satellite Sensors and Actuators using Nonlinear Geometric Approach and Adaptive Observers. International Journal of Robust and Nonlinear Control, 5429-5455. https://doi.org/10.1002/rnc.4083

\section{General rights}

Copyright and moral rights for the publications made accessible in the public portal are retained by the authors and/or other copyright owners and it is a condition of accessing publications that users recognise and abide by the legal requirements associated with these rights.

- Users may download and print one copy of any publication from the public portal for the purpose of private study or research.

- You may not further distribute the material or use it for any profit-making activity or commercial gain

- You may freely distribute the URL identifying the publication in the public portal 


\title{
Fault Diagnosis for Satellite Sensors and Actuators using Nonlinear Geometric Approach and Adaptive Observers
}

\author{
P. Baldi ${ }^{1 *}$, M. Blanke ${ }^{2}$, P. Castaldi ${ }^{1}$, N. Mimmo ${ }^{1}$ and S. Simani ${ }^{3}$ \\ ${ }^{1}$ Dipartimento di Ingegneria dell'Energia Elettrica e dell'Informazione, Università di Bologna, Facoltà di Ingegneria \\ Aerospaziale. 47100 Forli ( FC), Italy. \\ ${ }^{2}$ Department of Electrical Engineering, Technical University of Denmark, 2800 Kgs. Lyngby, Denmark. \\ ${ }^{3}$ Dipartimento di Ingegneria, Università di Ferrara. 44123 Ferrara (FE), Italy.
}

\begin{abstract}
SUMMARY
This paper presents a novel scheme for diagnosis of faults affecting sensors that measure the satellite attitude, body angular velocity, flywheel spin rates, and defects in control torques from reaction wheel motors. The proposed methodology uses adaptive observers to provide fault estimates that aid detection, isolation and estimation of possible actuator and sensor faults. The adaptive observers do not need a-priori information about fault internal models. A nonlinear geometric approach is used to avoid that aerodynamic disturbance torques have unwanted influence on the fault estimates. An augmented high fidelity spacecraft model is exploited during design and validation to replicate faults. This simulation model includes disturbance torques as experienced in low Earth orbits. The paper includes an analysis to assess robustness properties of the method with respect to parameter uncertainties and disturbances. The results document the efficacy of the suggested methodology.
\end{abstract}

Received ...

KEY WORDS: fault diagnosis; nonlinear geometric approach; adaptive observer; structural analysis; actuators and sensors.

\section{INTRODUCTION}

The increasing operational requirements for onboard autonomy in satellite control systems imply an inherent need for structural methods that support the design of complete and reliable supervisory systems. It is necessary to design supervision schemes that are capable of realising accurate diagnosis of potential faults, in order to allow subsequent fault accommodation actions to improve system reliability and availability, while maintaining desirable performances. In this context, Fault Detection and Diagnosis (FDD) systems provide fundamental information about the health status of the system jointly with the estimation of any faults.

Significant research in FDD has been done in last three decades $[1,2]$ and numerous model-based methods have been proposed [3, 4]. For a nonlinear spacecraft, linear models fall short, so nonlinear approaches are needed [5, 6]. The NonLinear Geometric Approach (NLGA) [7] was inspired by the Fault Detection and Isolation (FDI) problem for spacecraft. Later research $[8,9,10]$ investigated FDI and FDD methods for spacecraft, and some included supervisory actions to mitigate faults. Specificly, [11] considered faults in reaction wheel control torque and in wheel spin rate sensors, and [12] mapped physical faults to models that were affine with respect to actuator and sensor faults. The FDI task was carried out, in [11, 13], through cross-checking of residual signals and actuator and sensor fault estimates were provided by dedicated estimation filters using Radial Basis

\footnotetext{
${ }^{*}$ Correspondence to: Dipartimento di Ingegneria dell'Energia Elettrica e dell'Informazione, Università di Bologna, Facoltà di Ingegneria Aerospaziale. 47100 Forlì(FC), Italy. E-mail: pietro.baldi2@unibo.it
} 
Function Neural Networks (RBF NN). This approach was quite complex and robustness properties were not investigated. The majority of results in spacecraft FDD literature focus on occurrence of either actuator or sensor defects separately (se e.g. $[14,15,16]$ ) or take into account the possible occurrence of a more limited number of actuator and sensor faults at the same time, a more holistic view is needed on diagnosis and operability of the entire ADCS.

This paper aims to assess the health condition and proper functioning of all essential sensors and actuators used in a spacecraft Attitude Determination and Control System (ADCS). In particular, the sensors measuring the satellite attitude, body angular velocity and flywheel spin rates are considered, whereas the actuators are the reaction wheel motors. This work represents a substantial improvement over previous works of the authors [11,13]. Reduction in complexity is achieved by a structure where fault estimates from Adaptive Observers (AO) are used as diagnostic signals for all of the fault detection, isolation and estimation tasks. Step-stones in the design are based on the general works $[17,18]$. One is to augment models for the design of adaptive observers for sensor fault estimation, such that actual output sensor faults are represented as input signals [19]. The second is the design of adaptive observers for actuator and sensor fault estimation. A third is to employ the NLGA [7] to obtain diagnostic signals that are independent of the knowledge of the aerodynamic disturbance parameters and decoupled from different subsets of faults. The fourth is to achieve fault isolation through the use of a cross-check of the diagnostic signals and a proper decision logic.

Albeit being based on existing theoretical approaches, this paper describes a novel application scheme with significant benefits. The joint use of the NLGA and adaptive observers allows to take advantage of the benefits of both of them. The use of adaptive observers allows to design generalised fault estimation filters which do not need a priori information about the type of fault. The FDD adaptive observers can accurately estimate a generic fault without needing to define any specific fault internal model. The NLGA allows to obtain better FDI performances and accurate fault estimates, independent of the knowledge of the aerodynamic disturbance parameters, and thus without any isolation and estimation error due to aerodynamic parameter uncertainties. The Structural Analysis (SA) method, which is illustrated in [1] and already suggested for satellite applications for example in [20,21], is exploited to qualitatively assess detectability and isolability of faults related to the satellite attitude control system. This satellite-wide analysis of the ADCS will show that a second physical attitude sensor is required to achieve a complete isolation of possible attitude and angular velocity sensor faults.

The proposed scheme relies on general satellite and reaction wheel dynamic models and a very limited sensor hardware redundancy, and exploits only sensors and models that are fundamental for the ADCS. No additional subsystem models or embedded measurement sensors, e.g. current or voltage sensors, are required to achieve a complete FDD. Moreover, in practice, it is normal and usually required for safety and reliability reasons to have hardware redundancy available for both for satellite actuators and sensor systems.

The paper evaluates the performance of the proposed FDD system using a detailed nonlinear satellite simulator with detailed flywheel modeling [24], measurement noise and exogenous disturbance signals. In particular, the exogenous disturbance terms are represented by aerodynamic and gravitational disturbance torques. Simulation results show various fault cases. An extensive MonteCarlo analysis is conducted to assess the robustness and reliability of the proposed diagnosis scheme with respect to parametric uncertainties.

The paper is organised as follows. Section 2 describes the overall spacecraft and reaction wheel models. Section 3 illustrates the structural analysis of the actuator and sensor fault detectability and isolability. Section 4 illustrates the augmented spacecraft model. Section 5 illustrates the design of the FDD system, which is based on NLGA and adaptive observers. Section 6 illustrates the proposed procedure for the cross-checking of the diagnostic signals and the detection and isolation of the actuator or sensor faults. Section 7 provides simulation results. Concluding remarks are finally drawn in Section 8. 


\section{SPACECRAFT AND ACTUATOR MODELS}

\subsection{Dynamic and Kinematic Equations}

The spacecraft is considered as a rigid body, whose attitude is represented by using the quaternion notation. The satellite mathematical model is given by the dynamic and kinematic equations of (1) and (2) [22, 23], where $\omega_{\text {in }}=\left[\omega_{1}, \omega_{2}, \omega_{3}\right]_{i n}^{T}$ is the vector of body rates in roll, pitch, and yaw with respect to the inertial reference frame, respectively, whilst $\mathbf{h}_{r w}=\left[h_{r w, 1}, h_{r w, 2}, h_{r w, 3}, h_{r w, 4}\right]^{T}$ is the vector of the flywheel angular momenta. The quaternion vector qorb $=\left[q_{1}, q_{2}, q_{3}, q_{4}\right]_{\text {orb }}^{T}$ describes the attitude of the spacecraft with respect to the orbital reference frame. The principal inertia body-fixed frame is considered, with $I_{x x}, I_{y y}$, and $I_{z z}$, the elements on the main diagonal of the satellite inertia matrix $\mathbf{I}_{s}$.

$$
\begin{gathered}
\dot{\omega}_{\text {in }}=-\mathbf{I}_{s}^{-1} \mathbf{S}\left(\mathbf{I}_{s} \omega_{\text {in }}+\mathbf{T}_{r w} \mathbf{h}_{r w}\right)+\mathbf{I}_{s}^{-1}\left(-\mathbf{T}_{r w} \dot{\mathbf{h}}_{r w}+\mathbf{M}_{g g}+\mathbf{M}_{a e r o}\right) \\
\dot{\mathbf{q}}_{\text {orb }}=\frac{1}{2} \boldsymbol{\Omega} \mathbf{q}_{\text {orb }}
\end{gathered}
$$

with the skew-symmetric matrices

$$
\mathbf{S}\left(\omega_{\text {in }}\right)=\left[\begin{array}{ccc}
0 & -\omega_{3} & \omega_{2} \\
\omega_{3} & 0 & -\omega_{1} \\
-\omega_{2} & \omega_{1} & 0
\end{array}\right]_{\text {in }}, \boldsymbol{\Omega}\left(\omega_{\text {orb }}\right)=\left[\begin{array}{cccc}
0 & \omega_{3} & -\omega_{2} & \omega_{1} \\
-\omega_{3} & 0 & \omega_{1} & \omega_{2} \\
\omega_{2} & -\omega_{1} & 0 & \omega_{3} \\
-\omega_{1} & -\omega_{2} & -\omega_{3} & 0
\end{array}\right]_{\text {orb }}
$$

The relations between the spacecraft angular rates $\omega_{\text {in }}=\left[\omega_{1}, \omega_{2}, \omega_{3}\right]_{\text {in }}^{T}$ and $\omega_{\text {orb }}=\left[\omega_{1}, \omega_{2}, \omega_{3}\right]_{\text {orb }}^{T}$, respectively expressed with respect to the inertial and orbital frames, are given by

$$
\left[\begin{array}{l}
\omega_{1} \\
\omega_{2} \\
\omega_{3}
\end{array}\right]_{\text {orb }}=\left[\begin{array}{l}
\omega_{1} \\
\omega_{2} \\
\omega_{3}
\end{array}\right]_{\text {in }}+\omega_{0}\left[\begin{array}{c}
2\left(q_{1} q_{2}+q_{3} q_{4}\right) \\
\left(1-2 q_{1}^{2}-2 q_{3}^{2}\right) \\
2\left(q_{2} q_{3}-q_{1} q_{4}\right)
\end{array}\right]
$$

where $\omega_{0}=\sqrt{\mu / R^{3}}$ is the orbital angular velocity of the spacecraft. Therefore, it results that the dynamic equations of (1) describe the spacecraft dynamics with respect to the inertial reference frame, whilst the kinematic equations of (2) describe the attitude kinematics with respect to the orbital reference frame. The inertial and orbital notations for the spacecraft angular velocity and attitude, respectively, will be neglected in the rest of the paper.

The external disturbances, which can be cyclic or constant, consist of aerodynamic effects, gravity gradient, magnetic moment, and solar effects. In this paper, the design of the FDD system exploits the explicit decoupling only of the aerodynamic torque for the following reasons:

- the gravity is always present and, in many cases, produces disturbance torque at least one order of magnitude larger than other external torques (e.g. Galileo, Voyager 1 and 2). However, its model is almost perfectly known, and thus its effect does not need to be decoupled;

- many Low Earth Orbit (LEO) spacecrafts are not subject to significant solar disturbance torques;

- it is desirable to have a spacecraft with no on-board residual magnetic field that affects attitude; but there are cases in which magnetic actuators are used for attitude control purpose (control torque is known).

The dynamic equations (1) explicitly include the gyroscopic terms due to cross-couplings between the satellite angular rates and flywheel spin rates and the models of the gravitational and aerodynamic disturbance torques $\mathbf{M}_{g g}$ and $\mathbf{M}_{a e r o}$ about the centre of mass and described in the body-fixed frame. Both the disturbances are dependant on the satellite attitude. As already remarked, these disturbances typically represent the most important external disturbance torques affecting LEO satellites $[22,23]$. 
Regarding the gravity gradient torque $\mathbf{M}_{g g}$, the parameters $\mu$ and $R$ in (5) represent the gravitational constant and the orbit radius respectively. $R$ is the satellite distance from the Earth center and $\hat{v}_{\text {nadir }}$ is the unit vector towards nadir expressed in body-frame coordinates.

$$
\mathbf{M}_{g g}=\frac{3 \mu}{R^{3}}\left(\hat{v}_{\text {nadir }} \times I_{s} \hat{v}_{n a d i r}\right)=\frac{3 \mu}{R^{3}}\left[\begin{array}{c}
2\left(I_{z z}-I_{y y}\right)\left(q_{2} q_{3}+q_{1} q_{4}\right)\left(1-2 q_{1}^{2}-2 q_{2}^{2}\right) \\
2\left(I_{x x}-I_{z z}\right)\left(q_{1} q_{3}-q_{2} q_{4}\right)\left(1-2 q_{1}^{2}-2 q_{2}^{2}\right) \\
4\left(I_{y y}-I_{x x}\right)\left(q_{1} q_{3}-q_{2} q_{4}\right)\left(q_{2} q_{3}+q_{1} q_{4}\right)
\end{array}\right]
$$

Regarding the aerodynamic torque $\mathbf{M}_{\text {aero }}$, it depends on the aerodynamic force represented in (6) by the relation $F_{\text {aero }}=\frac{1}{2} \rho S_{p} V^{2} C_{D}$, where $\rho$ is the atmospheric density, $V$ is the relative velocity of the satellite, $S_{p}$ is the reference area affected by the aerodynamic flux, and $C_{D}$ is the drag coefficient. $\mathbf{r}_{\mathbf{c p}}=\left[r_{x_{c p}}, r_{y_{c p}}, r_{z_{c p}}\right]^{T}$ is the vector joining the centre of mass and the aerodynamic centre of pressure and $\hat{v}_{V}$ is the unit velocity vector expressed in body-frame coordinates. It is worth noting that, mainly due to the presence of the unknown terms $\rho$ and $C_{D}$, the input signal $\mathbf{M}_{\text {aero }}$ in (1) represents the main source of uncertainty. Therefore, the adaptive observers need to be independent of the aerodynamic disturbance. The aerodynamic torque is:

$$
\mathbf{M}_{\text {aero }}=F_{\text {aero }}\left(\hat{v}_{V} \times \mathbf{r}_{c p}\right)=\frac{1}{2} \rho S_{p} V^{2} C_{D}\left[\begin{array}{c}
2\left(q_{1} q_{3}+q_{2} q_{4}\right) r_{y_{c p}}-2\left(q_{1} q_{2}-q_{3} q_{4}\right) r_{z_{c p}} \\
\left(1-2 q_{2}^{2}-2 q_{3}^{2}\right) r_{z_{c p}}-2\left(q_{1} q_{3}+q_{2} q_{4}\right) r_{x_{c p}} \\
2\left(q_{1} q_{2}-q_{3} q_{4}\right) r_{x_{c p}}-\left(1-2 q_{2}^{2}-2 q_{3}^{2}\right) r_{y_{c p}}
\end{array}\right]
$$

The considered satellite Attitude Control System (ACS) consists of a fixed array of four reaction wheels in a tetrahedral configuration defined by the matrix $\mathbf{T}_{r w}$ :

$$
\mathbf{T}_{r w}=\left[\begin{array}{cccc}
1 / \sqrt{3} & 1 / \sqrt{3} & -1 / \sqrt{3} & -1 / \sqrt{3} \\
\sqrt{2 / 3} & -\sqrt{2 / 3} & 0 & 0 \\
0 & 0 & -\sqrt{2 / 3} & \sqrt{2 / 3}
\end{array}\right]
$$

The dynamic equations of the detailed reaction wheel models are given in (8), where $\mathbf{h}_{r w}=$ $I_{r w} \omega_{r w}=\left[h_{r w, 1}, h_{r w, 2}, h_{r w, 3}, h_{r w, 4}\right]^{T}$ is the vector of the wheel angular momenta, $\omega_{r w}=$ $\left[\omega_{r w, 1}, \omega_{r w, 2}, \omega_{r w, 3}, \omega_{r w, 4}\right]^{T}$ is the vector of the reaction wheel spin rates, $I_{r w}$ denotes the flywheel inertia and $b, c$ are the viscous and Coulomb friction coefficients, respectively [24]:

$$
\dot{\omega}_{r w}=I_{r w}{ }^{-1} \dot{\mathbf{h}}_{r w}=I_{r w}{ }^{-1} \mathbf{M}_{\mathbf{r w}}-I_{r w}{ }^{-1} b \omega_{r w}-I_{r w}{ }^{-1} \operatorname{csgn} \omega_{r w}
$$

The elements of the input vector $\mathbf{u}=\mathbf{M}_{\mathbf{r w}}=\left[M_{1}, M_{2}, M_{3}, M_{4}\right]^{T}$ correspond to the control torques actuated by the reaction wheel motors.

The overall system model can be described by (1), (2) and (8). Thus, the overall state vector can be represented by $\mathbf{x}=\left[\omega_{1}, \omega_{2}, \omega_{3}, q_{1}, q_{2}, q_{3}, q_{4}, \omega_{r w, 1}, \omega_{r w, 2}, \omega_{r w, 3}, \omega_{r w, 4}\right]^{T}$, and all the state variables are assumed to be measurable.

\subsection{Actuator and Sensor Fault Modelling}

The occurrence of possible faults affecting the actuated attitude control torques, the flywheel spin rate, satellite attitude and angular velocity measurements is considered in this paper. Moreover, it is assumed that at most one fault can affect the system at any time. However, it is worth observing that the designed fault diagnosis system is able to detect, isolate and estimate any occurred actuator or sensor fault at any time without needing any reconfiguration of the diagnosis system on the basis of the considered fault. Being (1) and(8) already affine in the control inputs, the $i$-th physical actuator fault can be simply modeled through the following additive fault input:

$$
f_{M_{i}}=M_{i}-M_{c, i},(i=1, \ldots, 4)
$$

where $M_{c}$ represents the vector of the commanded control inputs. Regarding the sensor faults, the measurement faults can be defined as the differences between the real values $\omega_{r w, j}, \omega_{l}, q_{m}$ and 
measured values $\omega_{r w_{y, j}}, \omega_{y, l}, q_{y, m}$ of the $j$-th flywheel spin rate, $l$-th satellite angular velocity and $m$-th quaternion component, respectively:

$$
\begin{array}{ll}
f_{\omega_{r w, j}}=\omega_{r w_{y, j}}-\omega_{r w, j} & (j=1, \ldots, 4) \\
f_{\omega_{l}}=\omega_{y, l}-\omega_{l} & (l=1, \ldots, 3) \\
f_{q_{m}}=q_{y, m}-q_{m} & (m=1, \ldots, 4)
\end{array}
$$

It is worth noting that physical attitude sensor faults generally can have an effect on all the components of the provided quaternion vectors simultaneously, thus each physical attitude sensor fault is actually considered as a single additive fault vector $\mathbf{f}_{\mathbf{q}}=\left[f_{q_{1}}, f_{q_{2}}, f_{q_{3}}, f_{q_{4}}\right]^{T}$ affecting the measurement of the quaternion vector $\mathbf{q}$ in the rest of the paper.

The presence of fault terms in the output equations of the system does not allow the direct exploitation of the NLGA as described in [7]. However, the exploitation of an augmented model, as described in [17] and in the following Section 4, allows to represent the actual output sensor faults as input faults in the augmented model, and subsequently makes the system model suitable for the exploitation of the NLGA.

It is worth noting that in this paper, only additive fault representations are considered, due to the requirement of nonlinear system models affine both in the inputs and faults for the exploitation of the Nonlinear Geometric Approach (NLGA). The multiplicative model is, usually, a natural way to model a wide variety of sensor and actuator faults, but cannot be used to represent more general component faults [25]. On the other hand, the additive faults representation is more general than the multiplicative one and can be used to model a wide class of faults, including sensor, actuator, and component faults [25]. In addition, the additive faults representation is more suitable for the design of FDI/FDD schemes because the faults are represented by one signal rather than by changes in the dynamic model of the system, as is the case with the multiplicative representation. Finally, for actuator faults, the equivalent multiplicative fault magnitude is needed for controller redesign, and this involves that actuator command is available. However, for actuator fault mitigation, we often choose to disregard actuators with faults and mitigate using remaining healthy actuators. The latter approach is fully supported by the diagnosis presented in this paper. Multiplicative faults can always be modelled in an equivalent but additive form. Both additive and multiplicative fault representations can be equivalently exploited to model a wide variety of abrupt, incipient, intermittent actuator and sensor faults due to different causes (e.g. mechanical, electrical, thermal, magnetic causes, etc.).

\section{STRUCTURAL ANALYSIS OF FAULT DETECTABILITY AND ISOLABILITY}

Before starting with the design of the fault diagnosis system, it is worth observing that the availability of two different attitude sensors has been actually considered in this work. This hardware redundancy is necessary for the complete sensor fault isolability and comes as outcome of the application of fault detectability and isolability study to the considered fault scenarios. A structural analysis has been performed as illustrated in [1]. For a comprehensive detailed application of the structural analysis, refer to [1].

The structural analysis has be applied to the spacecraft model (13), which can be interpreted as a set of constraints (i.e. a set of nominal input-output relations), to define a structure graph describing the direct interactions among the signals within the dynamical system and independently of the nature of these constraints. This graph gives a qualitative representation of the links between constraints and the variables and parameters occurring in each constraint and allows to analyse the redundancies within the system, which can be exploited for fault diagnosis.

The behavioural model of a system is defined by a pair $\mathcal{S}=(\mathcal{C}, \mathcal{Z})$ where $\mathcal{Z}=\left\{z_{1}, z_{2}, \ldots, z_{N}\right\}$ is a set of variables and parameters and $\mathcal{C}=\left\{c_{1}, c_{2}, \ldots, c_{M}\right\}$ is a set of constraints describing the relations among the variables. The structural model of the system $\mathcal{S}=(\mathcal{C}, \mathcal{Z})$ is a bipartite graph $\mathcal{G}=(\mathcal{C}, \mathcal{Z}, \mathcal{E})$ where $\mathcal{E} \subset \mathcal{C} \times \mathcal{Z}$ is the set of edges $\left(c_{i}, z_{j}\right) \in \mathcal{E}$ if the variable $z_{j}$ appears in the constraint $c_{i}$. In this undirected graph, all the variables and parameters $z_{j} \in \mathcal{Z}$ that are connected with a given constraint-vertex $c_{i} \in \mathcal{C}$ have to satisfy the equation or rule that this constraint-vertex represents. 
The system variables and parameters $\mathcal{Z}$ can be classified as known and unknown ones. Unknown variables $\mathcal{X}$ are not directly measured, though there might exist some way to compute their value from the values of known variables $\mathcal{K}$. Similarly, the set of constraints $\mathcal{C}$ can be partitioned into the subsets of constraints $\mathcal{C}_{\mathcal{K}}$, which link only known variables, and $\mathcal{C}_{\mathcal{X}}$, in which at least one unknown variable appears.

The basic tool for the structural analysis concerning fault detectability and isolability is the concept of matching in bipartite graphs $\mathcal{G}=(\mathcal{C}, \mathcal{Z}, \mathcal{E})$. A matching is a causal assignment, i.e. the introduction of some orientations of the originally undirected structure graph edges, which associates with every unknown system variable of $\mathcal{X}$ a constraint that can be used to determine the variable assuming the other variables of $\mathcal{Z}$ to be known. Unknown variables that do not appear in a matching cannot be calculated, whereas variables that can be matched in several ways can be determined in different (redundant) ways. The last situation provides a means for fault detection and isolation. A matching is called complete with respect to $\mathcal{C}$ if it has cardinality $|\mathcal{M}|=|\mathcal{C}|$, whilst it is called complete with respect to $\mathcal{X}$ if $|\mathcal{M}|=|\mathcal{X}|$ For a matching $\mathcal{M}$ that is complete with respect to $\mathcal{C}$, each constraint belongs to exactly one edge of the matching:

$$
\forall c \in \mathcal{C}: \exists x \in \mathcal{X} \text { suchthat }(c, x) \in \mathcal{M}
$$

Similarly, for a matching that is complete with respect to $\mathcal{X}$, every variable belongs to an edge:

$$
\forall x \in \mathcal{X}: \exists c \in \mathcal{C} \text { suchthat }(c, x) \in \mathcal{M}
$$

A graph $\mathcal{G}=(\mathcal{C}, \mathcal{Z}, \mathcal{E})$ is called over-constrained if there is a complete matching on the variables $\mathcal{X}$ but not on the constraints $\mathcal{C}$, just-constrained if there is a complete matching on the variables $\mathcal{X}$ and on the constraints $\mathcal{C}$ and under-constrained if there is a complete matching on the constraints $\mathcal{C}$ but not on the variables $\mathcal{X}$.

As illustrated in [1], a system is said to be structurally diagnosable or monitorable if it is possible to test whether the system constraints are satisfied or not. The analysis of system monitorability and the FDI algorithms are based on Analytical Redundancy Relations (ARR)s, which become available when there are constraints that are not needed to match the unknown variables in a system and some redundant information exists. These additional constraints, as well as all others, need be satisfied when the system operates according to its normal operation behaviour.

It is clear that ARRs can be defined only for over-constrained graphs, i.e. $\mathcal{X}$-complete matchings, because such matchings show a way to determine all the unknown variables of the system. The redundancy relations are identified as the unmatched constraints in which all the unknown variables have been matched, and subsequently the relations are expressed by known variables through backtracking to known variables, according to the matching.

Considering the occurrence of actuator or flywheel spin rate sensor faults $f_{M_{i}}$ and $f_{\omega_{r w, i}}(i=$ $1, \ldots, 4)$, each reaction wheel can be considered as a distinct subsystem. The obtainable graphs $\mathcal{G}_{i}=\left(\mathcal{C}_{i}, \mathcal{Z}_{i}, \mathcal{E}_{i}\right)(i=1, \ldots, 4)$ corresponding to the reaction wheel model equations (8) result to be over-constrained since the matching is complete on the unknown variables $\mathcal{X}_{i}$ but not on the constraints $\mathcal{C}_{i}$, i.e. $\left|\mathcal{M}_{i}\right|=\left|\mathcal{X}_{i}\right| \leq\left|\mathcal{C}_{i}\right|$. Therefore, it would be possible to define a distinct ARR for each reaction wheel subsystem from the resulting unmatched constraints.

However, since both actuator and sensor faults $f_{M_{i}}$ and $f_{\omega_{r w, i}}$ affecting the $i$-th reaction wheel subsystem are considered in this paper, it would not be possible to obtain the complete fault isolation only by means of these ARRs, but only the fault detection when the constraint is violated and the ARR not satisfied. In fact, each ARR results to be sensitive to both the actuator and sensor faults affecting a specific reaction wheel subsystem.

In order to allow the complete fault isolation, some additional constraints can be introduced to obtain an extended over-constrained graph by exploiting also the satellite dynamic equations (1). However, since in the considered spacecraft dynamic model (1) the aerodynamic disturbance is characterized by parameters whose actual values are generally not exactly known, the disturbance term $d=$ $\frac{1}{2} \rho S_{p} V^{2} C_{D}$ is considered as an unknown variable. As a consequence, the constraints associated with the equations (1) could be not satisfied even in case of no faults if the unknown aerodynamic parameters have actual values different from the assumed nominal ones. These discrepancies might 
lead to false alarms in the fault detection procedure. Therefore, the ARRs determined on the basis of these constraints would be not robust to aerodynamic parameter uncertainties.

As it will be shown in Section 5.4, the exploitation of the NLGA allows to define an additional mathematical variable $x_{a d d}$, whose dynamic equation $\dot{x}_{a d d}=f\left(\omega_{1}, \omega_{2}, \omega_{3}, \mathbf{q}, \omega_{r w, 1}, \omega_{r w, 2}, \omega_{r w, 3}, \omega_{r w, 4}\right)$ is exactly decoupled from the aerodynamic disturbance and any actuator fault $f_{M_{i}}(i=1, \ldots, 4)$, and generally sensitive to all the sensor faults, including any sensor fault $f_{\omega_{r w, i}}(i=1, \ldots, 4)$. $y_{\text {add }}$ represents the corresponding output variable. The constraints associated with this new variable and its dynamic and output equations allow to determine an additional ARR that is robust to the aerodynamic parameter uncertainties and exploitable for the complete isolation of faults $f_{M_{i}}$ or $f_{\omega_{r w, i}}(i=1, \ldots, 4)$ affecting any reaction wheel subsystem. Since the NLGA variable $x_{a d d}$ actually is not sensitive to any actuator fault and sensitive to any flywheel spin rate sensor fault, only the occurrence of any actual fault $f_{\omega_{r w, i}}(i=1, \ldots, 4)$ results in a violation of the constraints linked to $x_{\text {add }}$. The new graph is still over-constrained. Therefore, it would be possible to define five structured ARRs from the resulting unmatched constraints, which allow the complete detection and isolation of any actuator or flywheel spin rate sensor fault, without the risk of false alarms due to the aerodynamic parameter uncertainties.

On the other hand, considering the occurrence of satellite attitude and angular velocity sensor faults $f_{\omega_{l}}(l=1, \ldots, 3)$ and $\mathbf{f}_{\mathbf{q}}$ and the presence of a single attitude sensor, the kinematic equations (2) of the satellite attitude can be considered in the structural analysis. In this case, the corresponding graph $\mathcal{G}=(\mathcal{C}, \mathcal{Z}, \mathcal{E})$ results to be again over-constrained since the matching is complete on the unknown variables $\mathcal{X}$ but not on the constraints $\mathcal{C}$, i.e. $|\mathcal{M}|=|\mathcal{X}| \leq|\mathcal{C}|$. Therefore, it would be possible to define an ARR from the resulting unmatched constraint.

However, since the occurrence of both satellite attitude and angular velocity sensor faults $f_{\omega_{l}}$ $(l=1, \ldots, 3)$ and $\mathbf{f}_{\mathbf{q}}$ is now considered, it would not be possible to obtain the fault isolation only by means of a single ARR, but only the fault detection when the constraint is violated and the ARR is not satisfied. In fact, this ARR results to be sensitive to all the satellite attitude and angular velocity sensor faults $f_{\omega_{l}}(l=1, \ldots, 3)$ and $\mathbf{f}_{\mathbf{q}}$.

In order to allow the complete fault isolation, some additional constraints need again to be introduced. In this case, the equations describing the behaviour of the reaction wheel subsystems can not be exploited since they are functions only of the satellite attitude and angular velocities, and thus they are not sensitive to any fault affecting the satellite attitude or angular velocity sensors. Moreover, the NLGA variable $x_{a d d}$ previously introduced for the detection and isolation of actuator and flywheel spin rate sensor faults can not be effectively exploited since it leads to an additional ARR that is actually not satisfied when any sensor fault occurs. Hence, another way to determine additional ARRs to be exploited for the fault isolation task is necessary.

In order to determine structured ARRs exploitable for the complete isolation of attitude and angular velocity sensor faults, a two-steps procedure can be used. Firstly, the NLGA can be exploited to determine nine new mathematical variables $x_{a d d, i}(i=1, \ldots, 9)$, whose each dynamic equation $\dot{x}_{a d d, i}=f(\omega, \mathbf{q})(i=1, \ldots, 9)$ actually results to be decoupled from a specific angular velocity sensor fault $f_{\omega_{l}}(l=1, \ldots, 3)$ and sensitive to the couple of remaining angular velocity sensor faults and to the attitude sensor fault $\mathbf{f}_{\mathbf{q}} \cdot y_{a d d, i}$ represent the corresponding output variables. Further details about these NLGA variables are given in Section 5.5. The constraints associated with these new variables and their dynamic and output equations allow to determine ARRs that are structured with respect to the angular velocity sensor faults $f_{\omega_{l}}(l=1, \ldots, 3)$, thought they are still all sensitive to the attitude sensor fault $\mathbf{f}_{\mathrm{q}}$. Hence, in case of any angular velocity sensor fault, only a subset of ARRs is not satisfied, thus allowing to recognize the occurred angular velocity sensor fault. However, an additional ARR is still required in order to recognize if an attitude sensor fault or any angular velocity sensor fault actually occurred, and thus obtain the complete fault isolation.

Actually, the only practical way to determine this last ARR consists in the exploitation of some hardware sensor redundancy, which leads to the introduction of additional constraints on the unknown variables. In this paper, the presence of a redundant attitude sensor is then considered. The attitude measurements of these redundant sensors are represented in the following by means of two different quaternion vectors $\mathbf{q}_{\mathbf{y}, \mathbf{k}}(k=1,2)$, which are calculated on the basis of the information 
provided by two physical attitude sensors (e.g. star trackers). As a consequence, these attitude measurements can be affected by the corresponding sensor faults $\mathbf{f}_{\mathbf{q}, \mathbf{k}}(k=1,2)$.

Again, the corresponding graph $\mathcal{G}=(\mathcal{C}, \mathcal{Z}, \mathcal{E})$ results to be over-constrained since the matching is complete on the unknown variables $\mathcal{X}$ but not on the constraints $\mathcal{C}$, i.e. $|\mathcal{M}|=|\mathcal{X}| \leq|\mathcal{C}|$. Therefore, exploiting both the NLGA and an additional hardware attitude sensor redundancy, it is possible to define a sufficient number of ARRs from the resulting unmatched constraints, which allow the detection and complete isolation of any possible attitude sensor fault $\mathbf{f}_{\mathbf{q}, \mathbf{k}}(k=1,2)$ affecting one of the two redundant sensors or any angular velocity sensor fault $f_{\omega_{l}}(l=1, \ldots, 3)$.

\section{AUGMENTED NONLINEAR MODEL}

The overall nonlinear spacecraft model can be briefly written in the following form:

$$
\left\{\begin{array}{l}
\dot{x}=n(x)+g(x) u+\ell_{a}(x) f_{a}+p(x) d \\
y=h(x)+\ell_{s}(x) f_{s}
\end{array}\right.
$$

in which the state vector is $x(t) \in \mathcal{X}$ (an open subset of $\mathcal{R}^{n}$ ), $u(t) \in \mathcal{R}^{m}$ is the nominal control input vector, $f_{a}(t) \in \mathcal{R}^{h}$ and $f_{s}(t) \in \mathcal{R}^{q}$ are the actuator and sensor fault vectors, respectively, whilst $d(t) \in \mathcal{R}^{r}$ is the disturbance vector, and $y \in \mathcal{R}^{p}$ is the output vector. $n(x)$, the columns of $\ell_{a}(x)$, $\ell_{s}(x), g(x)$ and $p(x)$ are smooth vector fields, and $h(x)$ is a smooth map. In particular, for the complete spacecraft model, embedding also the reaction wheel models, the following vectors are defined:

$$
\begin{aligned}
& x=\left[\omega_{1}, \omega_{2}, \omega_{3}, \mathbf{q}, \omega_{r w, 1}, \omega_{r w, 2}, \omega_{r w, 3}, \omega_{r w, 4}\right]^{T} \\
& u=\left[M_{c, 1}, M_{c, 2}, M_{c, 3}, M_{c, 4}\right]^{T} \\
& d=F_{\text {aero }}=\frac{1}{2} \rho S_{p} V^{2} C_{D} \\
& f_{a}=\left[f_{M_{1}}, f_{M_{2}}, f_{M_{3}}, f_{M_{4}}\right]^{T} \\
& f_{s}=\left[f_{\omega_{1}}, f_{\omega_{2}}, f_{\omega_{3}}, \mathbf{f}_{\mathbf{q}, \mathbf{1}}, \mathbf{f}_{\mathbf{q}, \mathbf{2}}, f_{\omega_{r w, 1}}, f_{\omega_{r w, 2}}, f_{\omega_{r w, 3}}, f_{\omega_{r w, 4}}\right]^{T} \\
& y=\left[\omega_{y, 1}, \omega_{y, 2}, \omega_{y, 3}, \mathbf{q}_{\mathbf{y}, \mathbf{1}}, \mathbf{q}_{\mathbf{y}, \mathbf{2}}, \omega_{r w, 1}, \omega_{r w, 2}, \omega_{r w, 3}, \omega_{r w, 4}\right]^{T}
\end{aligned}
$$

with all the state variables assumed to be measurable,

$$
h(x)=\ell_{s}(x)=\left[\begin{array}{ccc}
I_{3} & 0 & 0 \\
0 & I_{4} & 0 \\
0 & I_{4} & 0 \\
0 & 0 & I_{4}
\end{array}\right]
$$

due to the considered attitude sensor redundancy and the terms $n(x), g(x), \ell_{a}(x)$ and $p(x)$ derived from the equations of (1), (2) and (8).

Considering the approach proposed in [17] for the diagnosis of sensor faults, the spacecraft model can be augmented by adding new state variables $z=\int_{0}^{t} y(\tau) d \tau$ corresponding to the integrated output variables, so that $\dot{z}(t)=h(x)+\ell_{s}(x) f_{s}$. The augmented system model with the new state variables $z$ and the corresponding new output variables $w$ is therefore given as

$$
\left\{\begin{aligned}
\dot{x} & =n(x)+g(x) u+\ell_{a}(x) f_{a}+p(x) d \\
\dot{z} & =h(x)+\ell_{s}(x) f_{s} \\
w & =z
\end{aligned}\right.
$$

or more synthetically as

$$
\begin{cases}\dot{\bar{x}} & =\bar{f}(\bar{x})+\bar{g}(\bar{x}) u+\bar{\ell}(\bar{x}) f+\bar{p}(\bar{x}) d \\ \bar{y}=w & =\bar{h}(\bar{x})\end{cases}
$$

where, for the considered spacecraft model, it results

$$
\begin{aligned}
\bar{x} & =\left[x^{T}, z^{T}\right]^{T} \\
z & =\left[z_{\omega_{1}}, z_{\omega_{2}}, z_{\omega_{3}}, z_{\mathbf{q}_{1}}, z_{\mathbf{q}_{2}}, z_{\omega_{r w, 1}}, z_{\omega_{r w, 2}}, z_{\omega_{r w}, 3}, z_{\omega_{r w, 4}}\right]^{T} \\
f & =\left[f_{a}^{T}, f_{s}^{T}\right]^{T}
\end{aligned}
$$




$$
\begin{gathered}
\bar{f}(\bar{x})=\left[\begin{array}{l}
n(x) \\
h(x)
\end{array}\right], \bar{g}(\bar{x})=\left[\begin{array}{c}
g(x) \\
0
\end{array}\right], \bar{h}(\bar{x})=\left[\begin{array}{ll}
0 & I_{15}
\end{array}\right] \\
\bar{\ell}(\bar{x})=\left[\begin{array}{cc}
\ell_{a}(x) & 0 \\
0 & \ell_{s}(x)
\end{array}\right], \bar{p}(\bar{x})=\left[\begin{array}{c}
p(x) \\
0
\end{array}\right]
\end{gathered}
$$

where $z_{\omega_{l}}(l=1, \ldots, 3)$, the vectors $z_{\mathbf{q}_{\mathbf{k}}}(k=1,2), z_{\omega_{r w, j}}(j=1, \ldots, 4)$ correspond to the integrated output variables $\int_{0}^{t} \omega_{y, l}(\tau) d \tau, \int_{0}^{t} \mathbf{q}_{\mathbf{y}, \mathbf{k}}(\tau) d \tau, \int_{0}^{t} \omega_{r w_{y, j}}(\tau) d \tau$ of the $l$-th satellite angular velocity measurement, $k$-th quaternion vector measurement and $j$-th flywheel spin rate measurement, respectively. It can be seen that the augmented model (17) of the spacecraft now results to be affine in all the control inputs and both actuator and sensor fault inputs. Thus, the NLGA can be exploited to design aerodynamic and fault decoupled adaptive observers also for the sensor fault diagnosis, as described in Section 5.

\section{FAULT DIAGNOSIS}

\subsection{Nonlinear Geometric Approach}

The NonLinear Geometric Approach was formally developed in [7], and it relies on a coordinate change in the state and output spaces providing an observable subsystem which, if it exists, is affected by the fault, but unaffected by disturbances and the other faults to be decoupled. The NLGA estimation filters for FDD are designed by exploiting the properties of this subsystem. For a comprehensive detailed application of the NLGA, refer to [7].

In particular, the approach consider a generic nonlinear system model in the form

$$
\left\{\begin{array}{l}
\dot{x}=n(x)+g(x) u+\ell(x) f+p(x) d \\
y=h(x)
\end{array}\right.
$$

in which the state vector $x \in \mathcal{X}$ (an open subset of $\mathcal{R}^{\ell_{n}}$ ), $u(t) \in \mathcal{R}^{\ell_{u}}$ is the nominal control input vector, $f(t) \in \mathcal{R}$ is the fault, $d(t) \in \mathcal{R}^{\ell_{d}}$ the disturbance vector (including also the faults to be decoupled), and $y \in \mathcal{R}^{\ell_{m}}$ the output vector. $n(x), \ell(x)$, the columns of $g(x)$ and $p(x)$ are smooth vector fields, and $h(x)$ is a smooth map. Therefore, if $P$ represents the distribution spanned by the column of $p(x)$, the NLGA method can be devised as follows [7]:

1. determine the minimal conditioned invariant distribution containing $P$ (denoted with $\sum_{*}^{P}$ );

2. by using $\left(\sum_{*}^{P}\right)^{\perp}$ (i.e. the maximal conditioned invariant codistribution contained in $P^{\perp}$ ), determine the largest observability codistribution contained in $P^{\perp}$ (denoted with $\Omega^{*}$ );

3. if $\ell(x) \notin\left(\Omega^{*}\right)^{\perp}$, the design procedure can continue, otherwise the fault is not detectable;

4. whenever the previous condition is satisfied, it can be found a surjection $\Psi_{1}$ and a function $\Phi_{1}$ fulfilling $\Omega^{*} \cap \operatorname{span}\{d h\}=\operatorname{span}\left\{d\left(\Psi_{1} \circ h\right)\right\}$ and $\Omega^{*}=\operatorname{span}\left\{d\left(\Phi_{1}\right)\right\}$, respectively.

The functions $\Psi(y)$ and $\Phi(x)$ defined as

$$
\Psi(y)=\left(\begin{array}{c}
\bar{y}_{1} \\
\bar{y}_{2}
\end{array}\right)=\left(\begin{array}{c}
\Psi_{1}(y) \\
H_{2} y
\end{array}\right), \Phi(x)=\left(\begin{array}{c}
\bar{x}_{1} \\
\bar{x}_{2} \\
\bar{x}_{3}
\end{array}\right)=\left(\begin{array}{c}
\Phi_{1}(x) \\
H_{2} h(x) \\
\Phi_{3}(x)
\end{array}\right)
$$

are (local) diffeomorphisms, where $H_{2}$ is a selection matrix (i.e. a matrix in which any row has all 0 entries but one, which is equal to 1$) . \bar{x}_{1}=\Phi_{1}(x)$ represents the measured part of the state which is affected by $f$ and not affected by $d$, whilst $\bar{x}_{2}$ and $\bar{x}_{3}$ represent the measured and not measured part of the state, which are affected by $f$ and $d$. In many cases $\bar{x}_{3}$ it is not present. In the new (local) 
coordinate defined previously, the system is described by the relations:

$$
\left\{\begin{aligned}
\dot{\bar{x}}_{1}= & n_{1}\left(\bar{x}_{1}, \bar{x}_{2}\right)+g_{1}\left(\bar{x}_{1}, \bar{x}_{2}\right) u+\ell_{1}\left(\bar{x}_{1}, \bar{x}_{2}, \bar{x}_{3}\right) f \\
\dot{\bar{x}}_{2}= & n_{2}\left(\bar{x}_{1}, \bar{x}_{2}, \bar{x}_{3}\right)+g_{2}\left(\bar{x}_{1}, \bar{x}_{2}, \bar{x}_{3}\right) u+ \\
& +\ell_{2}\left(\bar{x}_{1}, \bar{x}_{2}, \bar{x}_{3}\right) f+p_{2}\left(\bar{x}_{1}, \bar{x}_{2}, \bar{x}_{3}\right) d \\
\dot{\bar{x}}_{3}= & n_{3}\left(\bar{x}_{1}, \bar{x}_{2}, \bar{x}_{3}\right)+g_{3}\left(\bar{x}_{1}, \bar{x}_{2}, \bar{x}_{3}\right) u+ \\
& +\ell_{3}\left(\bar{x}_{1}, \bar{x}_{2}, \bar{x}_{3}\right) f+p_{3}\left(\bar{x}_{1}, \bar{x}_{2}, \bar{x}_{3}\right) d \\
\bar{y}_{1}= & h\left(\bar{x}_{1}\right) \\
\bar{y}_{2}= & \bar{x}_{2}
\end{aligned}\right.
$$

with $\ell_{1}\left(\bar{x}_{1}, \bar{x}_{2}, \bar{x}_{3}\right) \neq 0$ not identically zero. Denoting $\bar{x}_{2}$ with $\bar{y}_{2}$ and considering it as an independent input, the $\bar{x}_{1}$-subsystem, which is affected by the single fault $f_{u}$ and decoupled from the disturbance vector $d$ (embedding also the other faults to be decoupled), can be defined as follows:

$$
\left\{\begin{array}{l}
\dot{\bar{x}}_{1}=n_{1}\left(\bar{x}_{1}, \bar{y}_{2}\right)+g_{1}\left(\bar{x}_{1}, \bar{y}_{2}\right) u+\ell_{1}\left(\bar{x}_{1}, \bar{y}_{2}, \bar{x}_{3}\right) f \\
\bar{y}_{1}=h\left(\bar{x}_{1}\right)
\end{array}\right.
$$

with $\ell_{1}\left(\bar{x}_{1}, \bar{y}_{2}, \bar{x}_{3}\right) \neq 0$ not identically zero. This subsystem is exploited for the design of the adaptive observers and residual filters for fault diagnosis purpose.

\subsection{Generic Adaptive Input Fault Estimation Filter}

An adaptive observer can be designed for state observation and estimation of generic faults on the basis of the work presented in [17]. Consider a generic nonlinear system described by

$$
\left\{\begin{array}{l}
\dot{\bar{x}}(t)=\bar{A} \bar{x}(t)+\bar{W} \bar{f}(\bar{x}(t), t)+\bar{B} u(t)+\bar{D} f_{a}(t) \\
\bar{y}(t)=\bar{C} \bar{x}(t)
\end{array}\right.
$$

where $\bar{x} \in \mathbb{R}^{\bar{n}}, u \in \mathbb{R}^{\bar{m}}$ and $\bar{y} \in \mathbb{R}^{\bar{p}}$ denotes, respectively, the vector of state variables, inputs and outputs, $f_{a} \in \mathbb{R}^{\bar{q}}$ is a not measurable vector which is considered as an additive term resulting from generic input faults. The nonlinear continuous term $\bar{f}(\bar{x}(t), t) \in \mathbb{R}^{\bar{j}}$ is assumed to be known. $\bar{A} \in \mathbb{R}^{\bar{n} \times \bar{n}}, \bar{B} \in \mathbb{R}^{\bar{n} \times \bar{m}}, \bar{C} \in \mathbb{R}^{\bar{p} \times \bar{n}}, \bar{D} \in \mathbb{R}^{\bar{p} \times \bar{q}}(\bar{p} \geq \bar{q})$, and $\bar{W} \in \mathbb{R}^{\bar{n} \times \bar{j}}$ are known constant matrices with $\bar{C}$ and $\bar{D}$ being of full rank. The following assumptions are made:

Assumption 1. For every complex number $s$ with nonnegative real part,

$$
\operatorname{rank}\left[\begin{array}{c}
s I_{\bar{n}}-\bar{A} \\
\bar{C}
\end{array}\right]=\bar{n}
$$

Assumption 2. The nonlinear term $\bar{f}(\bar{x}(t), t)$ is assumed to be known and Lipschitz about $\bar{x}$ uniformly, i.e. $\forall \bar{x}, \hat{\bar{x}} \in \mathbb{R}^{\bar{n}}$,

$$
\|\bar{f}(\bar{x}(t), t)-\bar{f}(\hat{\bar{x}}(t), t)\| \leq \mathcal{L}_{f}\|\bar{x}(t)-\hat{\bar{x}}(t)\|
$$

where $\mathcal{L}_{f}$ is the Lipschitz constant and assumed to be unknown.

Assumption 3. The fault vector $f_{a}$ and its derivative $\dot{f}_{a}$ satisfy the following norm bounded constraints:

$$
\left\|f_{a}\right\| \leq \rho_{a},\left\|\dot{f}_{a}\right\| \leq \rho_{a a}
$$

where $\rho_{a}$ and $\rho_{a a}$ are known positive constants.

Lemma 1. The pair $(\bar{A}, \bar{C})$ is observable if Assumption 1 holds.

This Lemma is directly derived from the Popov-Belevitch-Hautus (PBH) observability criterion. It follows from Lemma 1 that there exists a matrix $L \in \mathbb{R}^{\bar{n} \times \bar{p}}$ such that $\bar{A}-L \bar{C}$ is stable, and thus for any $Q>0$, the Lyapunov equation

$$
(\bar{A}-L \bar{C})^{T} P+P(\bar{A}-L \bar{C})=-Q
$$

has a unique solution $P=P^{T}>0$, where $P \in \mathbb{R}^{\bar{n} \times \bar{n}}$ is a symmetric positive definite matrix and $Q \in \mathbb{R}^{\bar{n} \times \bar{n}}$. 
Remark 1. It follows from Assumption 1 that the pair $(\bar{A}, \bar{C})$ is observable, which provides the necessary and sufficient condition for the existence of an observer for system (25). Assumption 2 states that the considered nonlinear system is Lipschitz. Many practical systems satisfy the Lipschitz condition, at least locally. In Assumption 2 the generic input fault $f_{a}$ is assumed to be nonzero and differentiable after its occurrence. This assumption is quite general either for constant faults and time-varying faults at limited rates.

Assumption 4. There exists an arbitrary matrix $F \in \mathbb{R}^{\bar{h} \times \bar{p}}$ such that

$$
\bar{D}^{T} P=F \bar{C}
$$

For system (25) an adaptive observer is proposed in the following form:

$$
\left\{\begin{array}{l}
\dot{\bar{x}}(t)=\bar{A} \hat{\bar{x}}(t)+\bar{W} \bar{f}(\hat{\bar{x}}, t)+\bar{B} u(t)+L(\bar{y}-\hat{\bar{y}})+\frac{1}{2} \hat{k} \bar{W} H(\bar{y}-\hat{\bar{y}})+\bar{D} \hat{f}_{a} \\
\hat{\bar{y}}=\bar{C} \hat{\bar{x}}
\end{array}\right.
$$

where the observer gain $L \in \mathbb{R}^{\bar{n} \times \bar{p}}, H \in \mathbb{R}^{\bar{j} \times \bar{p}}$ is a matrix to be determined and $\hat{k}$ satisfies the following adaptation law:

$$
\dot{\hat{k}}=l_{k}\|H(\bar{y}-\hat{\bar{y}})\|^{2}
$$

where $l_{k}$ is a positive constant.

The term $\hat{f}_{a}$ represents the estimated fault and its dynamics is defined as:

$$
\dot{\hat{f}}_{a}=\Gamma F(\bar{y}-\hat{\bar{y}})-\varepsilon \Gamma \hat{f}_{a}
$$

where $\Gamma \in \mathbb{R}^{\bar{h} \times \bar{h}}$ is a symmetric positive definite matrix representing the learning rate, $F \in \mathbb{R}^{\bar{h} \times \bar{p}}$ is a matrix to be determined and $\epsilon$ is a positive scalar.

Denote $e_{x}=\bar{x}-\hat{\bar{x}}, e_{y}=\bar{y}-\hat{\bar{y}}$ and $e_{f}=f_{a}-\hat{f}_{a}$. Then, after the occurrence of the fault, the dynamics of the state estimation error is obtained from

$$
\dot{e}_{x}=(\bar{A}-L \bar{C}) e_{x}+\bar{W}(\bar{f}(x, t)-\bar{f}(\hat{\bar{x}}, t))+\bar{D} e_{f}
$$

The following regions can be defined:

$$
\begin{aligned}
& \Omega_{1}=\left\{\left(e_{y}, \hat{f}_{a}\right) \mid \frac{\lambda_{\min }(P)}{\|\bar{C}\|^{2}}\left\|e_{y}\right\|^{2}+\frac{\lambda_{\min }\left(\Gamma^{-1}\right)}{2}\left\|\hat{f}_{a}\right\|^{2} \leq \lambda_{\min }\left(\Gamma^{-1}\right) \rho_{a}^{2}+\frac{\mu_{3}}{\mu_{6}}\right\} \\
& \Omega_{2}=\left\{\left(e_{y}, \hat{f}_{a}\right) \mid \frac{\lambda_{\min }(P)}{\|\bar{C}\|^{2}}\left\|e_{y}\right\|^{2}+\frac{\lambda_{\min }\left(\Gamma^{-1}\right)}{2}\left\|\hat{f}_{a}\right\|^{2}>\lambda_{\min }\left(\Gamma^{-1}\right) \rho_{a}^{2}+\frac{\mu_{3}}{\mu_{6}}\right\} \\
& \mu_{1}=\lambda_{\min }\left(-(\bar{A}-L \bar{C})^{T} P-P(\bar{A}-L \bar{C})-2 I_{\bar{n}}\right)>0 \\
& \mu_{2}=\lambda_{\min }(\varepsilon I-G)>0 \\
& \mu_{3}=\rho_{a a}^{2} \lambda_{\max }\left(\Gamma^{-1} G^{-1} \Gamma^{-1}\right)+\varepsilon \rho_{a}^{2} \\
& \mu_{4}=\min \left(\mu_{1}, \mu_{2}\right) \\
& \mu_{5}=\max \left(\lambda_{\max }(P), \lambda_{\max }\left(\Gamma^{-1}\right)\right) \\
& \mu_{6}=\mu_{4} / \mu_{5}
\end{aligned}
$$

where $G \in \mathbb{R}^{\bar{q} \times \bar{q}}, P=P^{T} \in \mathbb{R}^{\bar{n} \times \bar{n}}$ are symmetric positive definite matrices and $\lambda_{\min }$ and $\lambda_{\max }$ are the smallest and largest eigenvalues, respectively.

Theorem 1. Given system (25) with Assumptions 1, 2 and 3, if there exist matrices $L, F, H$ and $P=P^{T}>0$ such that

$$
\begin{gathered}
\bar{D}^{T} P=F \bar{C} \\
\bar{W}^{T} P=H \bar{C} \\
-Q+2 I_{\bar{n}}<0
\end{gathered}
$$

where $-Q=(\bar{A}-L \bar{C})^{T} P+P(\bar{A}-L \bar{C}) \in \mathbb{R}^{\bar{n} \times \bar{n}}$, then for a given matrix $\Gamma$ and a positive scalar $\epsilon$, the error dynamics (34) is uniformly bounded and $\left(e_{y}, \hat{f}_{a}\right)$ converges to $\Omega_{1}$ at a rate greater than $e^{-\mu_{6} t}[17]$. 
Proof

Consider the Lyapunov function as

$$
V=e_{x}^{T} P e_{x}+l_{k}^{-1} e_{k}^{2} / 2+e_{f}^{T} \Gamma^{-1} e_{f}
$$

where $e_{k}=k-\hat{k}, k$ is a constant which is defined as $k=\mathcal{L}_{f}^{2}$. The time derivative of $V$ can be shown to be

$$
\begin{aligned}
\dot{V} & \leq-e_{x}^{T} Q e_{x}+\left\|e_{x}\right\|^{2}+\left(\mathcal{L}_{f}^{2}-\hat{k}\right)\left\|H C e_{x}\right\|^{2}-e_{k}\left\|H \bar{C} e_{x}\right\|^{2}+2 e_{f}^{T} \Gamma^{-1} \dot{f}_{a}+2 \varepsilon e_{f}^{T} f_{a}-2 \varepsilon e_{f}^{T} e_{f} \\
& \leq e_{x}^{T}(-Q+2 I) e_{x}+2 e_{f}^{T} \Gamma^{-1} \dot{f}_{a}+2 \varepsilon e_{f}^{T} f_{a}-2 \varepsilon e_{f}^{T} e_{f}
\end{aligned}
$$

Since $2 X^{T} Y \leq \frac{1}{\alpha} X^{T} G X+\alpha Y^{T} G^{-1} Y$ holds for any scalar $\alpha>0$ and symmetric positive definite matrix $G$, therefore

$$
2 e_{f}^{T} \Gamma^{-1} \dot{f}_{a} \leq e_{f}^{T} G e_{f}+\dot{f}_{a}^{T} \Gamma^{-1} G^{-1} \Gamma^{-1} \dot{f}_{a} \leq e_{f}^{T} G e_{f}+\rho_{a a} \lambda_{\max }\left(\Gamma^{-1} G^{-1} \Gamma^{-1}\right)
$$

Moreover

$$
2 \varepsilon e_{f}^{T} f_{a} \leq \varepsilon\left\|e_{f}\right\|^{2}+\varepsilon \rho_{a}^{2}
$$

Substituting (41) and (42) in (40) gives

$$
\begin{aligned}
\dot{V} & \leq-e_{x}^{T}(Q-2 I) e_{x}+e_{f}^{T}(G-\varepsilon I) e_{f}+\rho_{a a} \lambda_{\max }\left(\Gamma^{-1} G^{-1} \Gamma^{-1}\right)+\varepsilon \rho_{a}^{2} \\
& \leq-\mu_{1}\left\|e_{x}\right\|^{2}-\mu_{2}\left\|e_{f}\right\|^{2}+\mu_{3} \\
& \leq-\mu_{4}\left(\left\|e_{x}\right\|^{2}+\left\|e_{f}\right\|^{2}\right)+\mu_{3}
\end{aligned}
$$

Moreover, from (39)

$$
\begin{aligned}
V & \leq \lambda_{\max }(P)\left\|e_{x}\right\|^{2}+\lambda_{\max }\left(\Gamma^{-1}\right)\left\|e_{f}\right\|^{2} \\
& \leq \max \left(\lambda_{\max }(P), \lambda_{\max }\left(\Gamma^{-1}\right)\right)\left(\left\|e_{x}\right\|^{2}+\left\|e_{f}\right\|^{2}\right) \\
& =\mu_{5}\left(\left\|e_{x}\right\|^{2}+\left\|e_{f}\right\|^{2}\right)
\end{aligned}
$$

Then

$$
\dot{V} \leq-\mu_{6} V+\mu_{3}
$$

For any real constant $\bar{p}$ and $\bar{q} \in \mathbb{R}$, it results that

$$
(\bar{p}-\bar{q})^{2} \geq \frac{\bar{p}^{2}}{2}-\bar{q}^{2}
$$

Therefore

$$
V \geq \lambda_{\min }(P)\left\|e_{x}\right\|^{2}+\lambda_{\min }\left(\Gamma^{-1}\right)\left\|e_{f}\right\|^{2} \geq \frac{\lambda_{\min }(P)}{\|C\|^{2}}\left\|e_{y}\right\|^{2}+\lambda_{\min }\left(\Gamma^{-1}\right)\left(\frac{\left\|\hat{f}_{a}\right\|^{2}}{2}-\rho_{a}^{2}\right)
$$

If $\left(e_{y}, \hat{f}_{a}\right) \in \Omega_{2}$, then $V>\mu_{3} / \mu_{6}$ and consequently $\dot{V}<0$. Therefore, it can be concluded that $\left(e_{y}, \hat{f}_{a}\right)$ is uniformly bounded and converges to $\Omega_{1}$ exponentially at a rate greater than $e^{-\mu_{6} t}$.

Remark 2. The problem of finding matrices $P=P^{T}, L, H$ and $F$ to simultaneously satisfy the inequality (38) and equalities (36) and (37) can be transformed into the following LMI optimization problem:

$$
\begin{gathered}
\text { minimize } \gamma_{1}+\gamma_{2} \text { subject to } P>0 \text { and } \\
P \bar{A}+\bar{A}^{T} P-Y \bar{C}-\bar{C}^{T} Y^{T}+2 I_{\bar{n}}<0 \\
{\left[\begin{array}{cc}
\gamma_{1} I_{\bar{q}} & \bar{D}^{T} P-F \bar{C} \\
\left(\bar{D}^{T} P-F \bar{C}\right)^{T} & \gamma_{1} I_{\bar{n}}
\end{array}\right]>0,\left[\begin{array}{cc}
\gamma_{2} I_{\bar{j}} & \bar{W}^{T} P-H \bar{C} \\
\left(\bar{W}^{T} P-H \bar{C}\right)^{T} & \gamma_{2} I_{\bar{n}}
\end{array}\right]>0}
\end{gathered}
$$

where $Y=P L$. 


\subsection{Adaptation of the Generic Adaptive Estimator Filter in case of Output Sensor Faults}

In a similar way, an adaptive observer can be designed for state observation and estimation of generic sensor faults on the basis of the work presented in [17]. Consider a generic nonlinear system described by

$$
\left\{\begin{array}{l}
\dot{x}(t)=A x(t)+W f(x, t)+B u(t) \\
y(t)=C x(t)+D f_{s}(t)
\end{array}\right.
$$

where $x \in \mathbb{R}^{n}, u \in \mathbb{R}^{m}$ and $y \in \mathbb{R}^{p}$ denotes, respectively, the vector of state variables, inputs and outputs, $\bar{f}_{s} \in \mathbb{R}^{\bar{q}}$ is a not measurable vector which is considered as an additive term resulting from output sensor faults. The nonlinear continuous term $f(x, t) \in \mathbb{R}^{j}$ is assumed to be known. $A \in \mathbb{R}^{n \times n}, B \in \mathbb{R}^{n \times m}, C \in \mathbb{R}^{p \times n}, D \in \mathbb{R}^{p \times q}(p \geq q)$, and $W \in \mathbb{R}^{n \times j}$ are known constant matrices with $D$ being of full column rank. The following assumptions are made:

Assumption 5. The nonlinear term $f(x, t)$ is assumed to be known and Lipschitz about $x$ uniformly, i.e. $\forall x, \hat{x} \in \mathbb{R}^{n}$,

$$
\|f(x(t), t)-f(\hat{x}(t), t)\| \leq \mathcal{L}_{f}\|x(t)-\hat{x}(t)\|
$$

where $\mathcal{L}_{f}$ is the Lipschitz constant and assumed to be unknown.

Assumption 6. The output sensor fault vector $f_{s}$ and its derivative $\dot{f}_{s}$ satisfy the following norm bounded constraints:

$$
\left\|f_{s}\right\| \leq \rho_{s},\left\|\dot{f}_{s}\right\| \leq \rho_{s s}
$$

where $\rho_{s}$ and $\rho_{s s}$ are known positive constants.

As described in [17], for system (49) a new state variable $z=\int_{0}^{t} y(\tau) d \tau$ can be defined so that $\dot{z}(t)=C x(t)+D f_{o}(t)$. An augmented system with the new state $z$ and output $w$ is therefore given as

$$
\left\{\begin{array}{l}
\dot{x}(t)=A x(t)+W f(x, t)+B u(t) \\
\dot{z}(t)=C x(t)+D \bar{f}_{o}(t) \\
w(t)=z(t)
\end{array}\right.
$$

or in matricial form as

$$
\left[\begin{array}{c}
\dot{x} \\
\dot{z}
\end{array}\right]=\left[\begin{array}{cc}
A & 0 \\
C & 0
\end{array}\right]\left[\begin{array}{l}
x \\
z
\end{array}\right]+\left[\begin{array}{c}
W f(x, t) \\
0
\end{array}\right]+\left[\begin{array}{c}
B \\
0
\end{array}\right] u+\left[\begin{array}{c}
0 \\
D
\end{array}\right] f_{s}
$$

This system can further be rewritten in a more compact form as

$$
\left\{\begin{array}{l}
\dot{\bar{x}}(t)=\bar{A} \bar{x}(t)+\bar{W} f(\bar{x}, t)+\bar{B} u(t)+\bar{D} f_{o}(t) \\
\bar{y}(t)=w(t)=\bar{C} \bar{x}(t)
\end{array}\right.
$$

where $\bar{x} \in \mathbb{R}^{n+p}, \bar{y}=w \in \mathbb{R}^{p}, \bar{A}=\left[\begin{array}{ll}A & 0 \\ C & 0\end{array}\right] \in \mathbb{R}^{(n+p) \times(n+p)}, \bar{B}=\left[\begin{array}{c}B \\ 0\end{array}\right] \in \mathbb{R}^{(n+p) \times m}, \bar{D}=$ $\left[\begin{array}{c}0 \\ D\end{array}\right] \in \mathbb{R}^{(n+p) \times q}, \bar{C}=\left[\begin{array}{cc}0 & I_{p}\end{array}\right] \in \mathbb{R}^{p \times(n+p)}, \bar{W}=\left[\begin{array}{c}W \\ 0\end{array}\right] \in \mathbb{R}^{(n+p) \times m}$. It can be noted that the original sensor fault affecting the system output is now modelled as an input fault in the augmented system 54. Therefore, the same filter design procedure described in Section 5.2 for generic input faults $f_{u}$ can be adapted and exploited in order to design an adaptive filter for the estimation of a generic output sensor fault $f_{o}$, where $\bar{n}=n+p, \bar{p}=p, \bar{m}=m, \bar{q}=q$ and $\bar{j}=j$.

\subsection{Design of Adaptive Filters for the Estimation of Reaction Wheel Actuator Faults and Flywheel Spin Rate Sensor Faults}

The NLGA FDD system is designed on the basis of a input affine nonlinear model structure (21) as described in [7]. With the assumption of a single actuator or sensor fault occurring at any time, it is possible to design distinct adaptive observers, which are specifically designed to accurately estimate a particular actuator or sensor fault. The provided fault estimates can be directly exploited 
as diagnostic signals for the FDI task.

Considering the occurrence of possible actuator faults $f_{M i}(i=1, \ldots, 4)$ and since the measurements of the flywheel spin rate sensors are assumed to be available, the NLGA can be exploited in order to design four scalar adaptive observers as described in Section 5.2 starting from actuator model (8). These adaptive observers provide accurate estimates of the actuator faults and result to be independent of the aerodynamic disturbance and satellite attitude and angular velocity sensor faults. The scalar state variables $\bar{x}$ of these four NLGA adaptive observers are defined as follows:

$$
\begin{array}{ll}
\bar{x}_{1}=I_{r w} \omega_{r w, 1} & \bar{x}_{3}=I_{r w} \omega_{r w, 3} \\
\bar{x}_{2}=I_{r w} \omega_{r w, 2} & \bar{x}_{4}=I_{r w} \omega_{r w, 4}
\end{array}
$$

Therefore, considering the generic nonlinear model (25) and the related adaptive observer model (31), the following terms can be defined for each observer:

$$
\begin{aligned}
& \bar{A}=-\frac{b}{I_{r w}}, \bar{B}=1, \bar{C}=1, \bar{D}=1, \bar{W}=1 \\
& f\left(\bar{x}_{i}, t\right)=-\operatorname{csgnn} \bar{x}_{i}, u_{i}=M_{i}, f_{a, i}=f_{M_{i}} \quad(i=1, \ldots, 4)
\end{aligned}
$$

where $b$ is the viscous friction coefficient, $c$ is the Coulomb friction coefficient and $I_{r w}$ is the flywheel inertia.

The model of each of these four adaptive observers for $i=1, \ldots, 4$ is given by

$$
\left\{\begin{array}{l}
\dot{\bar{x}}_{i}(t)=\bar{A} \hat{\bar{x}}_{i}(t)+\bar{W} f\left(\hat{\bar{x}}_{i}, t\right)+\bar{B} u_{i}(t)+L\left(\bar{y}_{i}-\hat{\bar{y}}_{i}\right)+\frac{1}{2} \hat{k} \bar{W} H\left(\bar{y}_{i}-\hat{\bar{y}}_{i}\right)+\bar{D} \hat{f}_{a, i} \\
\hat{\bar{y}}_{i}(t)=\bar{C} \hat{\bar{x}}
\end{array}\right.
$$

The term $\hat{f}_{a, i}$ represents the estimated actuator fault affecting the $i$-th reaction wheel motor.

Remark 3. The provided fault estimates $\hat{f}_{a, i}(i=1, \ldots, 4)$ are directly exploited as diagnostic signals $\xi_{i}(i=1, \ldots, 4)$ also for the FDI task.

On the other hand, considering the occurrence of possible flywheel spin rate sensor faults $f_{\omega_{r w, j}}$ $(j=1, \ldots, 4)$, starting from the augmented nonlinear spacecraft model (17), the NLGA can be exploited in order to design four vectorial adaptive observers as described in Section 5.3. These adaptive observers provide accurate estimates of the flywheel spin rate sensor faults and result to be independent of the aerodynamic disturbance and satellite attitude and angular velocity sensor faults. The augmented state vectors $\bar{x}$ of these four NLGA adaptive observers are defined as follows:

$$
\begin{array}{ll}
\bar{x}_{1}=\left[\omega_{r w, 1}, z_{\omega_{r w, 1}}\right]^{T} & \bar{x}_{3}=\left[\omega_{r w, 3}, z_{\omega_{r w, 3}}\right]^{T} \\
\bar{x}_{2}=\left[\omega_{r w, 2}, z_{\omega_{r w, 2}}\right]^{T} & \bar{x}_{4}=\left[\omega_{r w, 4}, z_{\omega_{r w, 4}}\right]^{T}
\end{array}
$$

where $\omega_{r w, j}(j=1, \ldots, 4)$ are the spin rates of the four flywheels and $z_{\omega_{r w, j}}(j=1, \ldots, 4)$ are the corresponding new state variables introduced through the model augmentation described in Section 4 by means of the integration of the output variables.

Therefore, considering the generic nonlinear model (49) and the related adaptive observer model (31), the following terms can be defined for each observer:

$$
\begin{aligned}
& \bar{A}=\left[\begin{array}{cc}
-\frac{b}{I_{r w}} & 0 \\
1 & 0
\end{array}\right], \bar{B}=\left[\begin{array}{c}
\frac{1}{I_{r w}} \\
0
\end{array}\right], \bar{C}=\left[\begin{array}{ll}
0 & 1
\end{array}\right], \bar{D}=\left[\begin{array}{l}
0 \\
1
\end{array}\right], \bar{W}=\left[\begin{array}{l}
1 \\
0
\end{array}\right] \\
& f\left(\bar{x}_{j}, t\right)=-\frac{c s g n \omega_{r w, i}}{I_{r w}}, u_{j}=M_{j}, f_{s j}=f_{\omega_{r w_{j}}} \\
& \quad(j=1, \ldots, 4)
\end{aligned}
$$

The model of each of these four adaptive observers for $j=1, \ldots, 4$ is given by:

$$
\left\{\begin{array}{l}
\dot{\bar{z}}_{j}(t)=\bar{A} \hat{\bar{z}}_{j}(t)+\bar{W} f\left(\hat{\bar{z}}_{j}, t\right)+\bar{B} u_{j}(t)+\bar{L}\left(\bar{y}_{j}-\hat{\bar{y}}_{j}\right)+\frac{1}{2} \hat{k} \bar{W} \bar{H}\left(w_{j}-\hat{w}_{j}\right)+\bar{D} \hat{f}_{s, j} \\
\hat{\bar{y}}=\hat{w}_{j}=\bar{C} \hat{\bar{x}}_{j}
\end{array}\right.
$$

In this case, the term $\hat{f}_{s, j}$ represents the estimated fault affecting the $j$-th flywheel spin rate sensor. 
Remark 4. The provided fault estimates $\hat{f}_{s, j}(j=1, \ldots, 4)$ are directly exploited as diagnostic signals $\xi_{i}(i=5, \ldots, 8)$ for the FDI task.

Remark 5. It is worth noting that, actually, each of the eight NLGA adaptive observers described above results to be sensitive to the couple of faults $f_{M_{i}}, f_{\omega_{r w, j}}(i=j)$, i.e. the actuator and flywheel spin rate sensor faults related to the same $i$-th reaction wheel, respectively.

In fact, the flywheel spin rate sensor fault $f_{\omega_{r w, j}}$ directly affects the residual signal $\bar{y}_{i}-\hat{\bar{y}}_{i}$ driving the actuator fault estimate of the adaptive filters relying on the variables (55). On the other hand, the actuator fault $f_{M_{i}}$ still indirectly affects, through the integration of the measured sensor outputs, the residual signal $\bar{y}_{j}-\hat{\bar{y}}_{j}$ driving the sensor fault estimate of the adaptive filters relying on the variables (58).

However, since each of these eight adaptive estimation filters is designed to provide accurate estimates with respect to the occurrence of a specific actuator or flywheel spin rate sensor fault, the provided signals $\hat{f}_{a, i}(i=1, \ldots, 4)$ result to be correct fault estimates only in case of actuator faults $f_{M_{i}}$, whilst they do not represent estimates of the actual faults in case of flywheel spin rate sensor faults $f_{\omega_{r w, j}}$. Analogously, the provided signals $\hat{f}_{s, j}(j=1, \ldots, 4)$ result to be correct fault estimates only in case of sensor faults $f_{\omega_{r w, j}}$, whilst they do not represent estimates of the actual faults in case of actuator faults $f_{M_{i}}$.

Therefore, in general, these estimates allow for the isolation of the reaction wheel subsystem affected by a possible actuator or sensor fault, but it could not be possible to achieve an exact and complete fault isolation by exploiting only these signals. This can be achieved, thanks to the NLGA, by designing and exploiting an additional residual filter in order to precisely classify a detected fault as an actuator or sensor fault with the assumption of a single actuator or sensor fault occurring at any time. The use of the NLGA results to be fundamental to design an additional residual filter that results to be decoupled from the aerodynamic disturbance in order to obtain a diagnostic signal not subject to detection errors due to parametric uncertainties of the aerodynamic disturbance model. This NLGA residual filter exploits also satellite attitude and angular speed measurements in addition to the flywheel spin rate measurements. The dynamic equation determined through the NLGA results to be insensitive to any possible actuator fault and sensitive to a mathematical combination of all the spacecraft sensor faults, and thus also to the flywheel spin rate sensor faults.

Starting from (24), a generic residual generator in filter form is modelled as follows:

$$
\left\{\begin{array}{l}
\dot{\xi}=n_{1}\left(\bar{y}_{1}, \bar{y}_{2}\right)+g_{1}\left(\bar{y}_{1}, \bar{y}_{2}\right) u_{c}+L\left(\bar{y}_{1}-\xi\right) \\
\varepsilon=\bar{y}_{1}-\xi
\end{array}\right.
$$

where $L>0$ is the gain of the asymptotically stable residual filter and $\varepsilon$ is the generated diagnostic signal. The state vector $\bar{x}$ of this additional residual generator, obtained by means of the NLGA on the basis of (13), is defined as follows:

$$
\bar{x}=\left[r_{x_{c p}}\left(I_{x x} \omega_{1}+\mathbf{T}_{1} I_{r w} \omega_{r w}\right)+r_{y_{c p}}\left(I_{y y} \omega_{2}+\mathbf{T}_{2} I_{r w} \omega_{r w}\right)+r_{z_{c p}}\left(I_{z z} \omega_{3}+\mathbf{T}_{3} I_{r w} \omega_{r w}\right)\right]
$$

where $\mathbf{T}_{1}, \mathbf{T}_{2}$ and $\mathbf{T}_{3}$ are the rows of the matrix $\mathbf{T}_{r w}, z_{\omega_{l}}(l=1, \ldots, 3)$.

Remark 6 . The provided residual signal $\varepsilon$ is exploited as additional diagnostic signal $\xi_{9}$ to precisely classify a detected fault as an actuator or flywheel spin rate sensor fault for the complete isolation. After the correct isolation of the occurred actuator or sensor fault, the corresponding accurate estimate is selected among the diagnostic signals $\xi_{1}, \ldots, \xi_{8}$.

\subsection{Design of Adaptive Filters for the Estimation of Satellite Attitude and Angular Velocity Sensor Faults}

Considering the occurrence of possible faults $\mathbf{f}_{\mathbf{q}, \mathbf{k}}(k=1,2)$ affecting the two considered attitude sensors, starting from the augmented nonlinear spacecraft model (17), the NLGA can be exploited in order to design two adaptive observers as described in Section 5.3. These adaptive observers provide accurate estimates of the attitude sensor fault vectors and result to be independent of the aerodynamic disturbance and actuator and sensor faults affecting the reaction wheel subsystems. 
It is worth noting that, since the measurements of two attitude sensors are assumed to be available, each observer exploits the measurements of a different attitude sensor, in addition to the shared angular velocity measurements.

The augmented state vectors $\bar{x}$ of these two NLGA adaptive observers are defined as follows:

$$
\bar{x}_{1}=\left[\mathbf{q}^{T}, z_{\mathbf{q}_{1}}^{T}\right]^{T}, \bar{x}_{2}=\left[\mathbf{q}^{T}, z_{\mathbf{q}_{2}}^{T}\right]^{T}
$$

where $\mathbf{q}$ is the quaternion state vector and $z_{\mathbf{q}_{\mathbf{k}}}(k=1,2)$ are the corresponding new state vectors introduced through the model augmentation described in Section 4 by means of the integration of the available output vectors provided by the two considered attitude sensors. Each of these two observer exploits the quaternion measurements $\mathbf{q}_{\mathbf{y}, \mathbf{k}}(k=1,2)$ of a specific $k$-th attitude sensor and provides the accurate estimate of the corresponding fault vector $\mathbf{f}_{\mathbf{q}, \mathbf{k}}=\left[f_{q_{1}}, f_{q_{2}}, f_{q_{3}}, f_{q_{4}}\right]^{T}$ (with $k=1,2$ ). Therefore, considering the generic nonlinear model (49) and the related adaptive observer model (31), the following terms can be defined for each observer:

$$
\begin{gathered}
\bar{A}=\left[\begin{array}{cccccccc}
0 & 0 & \frac{\omega_{0}}{2} & 0 & 0 & 0 & 0 & 0 \\
0 & 0 & 0 & \frac{\omega_{0}}{2} & 0 & 0 & 0 & 0 \\
-\frac{\omega_{0}}{2} & 0 & 0 & 0 & 0 & 0 & 0 & 0 \\
0 & -\frac{\omega_{0}}{2} & 0 & 0 & 0 & 0 & 0 & 0 \\
1 & 0 & 0 & 0 & 0 & 0 & 0 & 0 \\
0 & 1 & 0 & 0 & 0 & 0 & 0 & 0 \\
0 & 0 & 1 & 0 & 0 & 0 & 0 & 0 \\
0 & 0 & 0 & 1 & 0 & 0 & 0 & 0
\end{array}\right], \bar{C}=\left[\begin{array}{ll}
0 & I_{4}
\end{array}\right], \bar{D}=\left[\begin{array}{c}
0 \\
I_{4}
\end{array}\right], \bar{W}=\frac{1}{2}\left[\begin{array}{c}
I_{4} \\
0
\end{array}\right] \\
f\left(\bar{z}_{k}, y, t\right)=\left[\begin{array}{c}
\omega_{3} q_{2}-\omega_{2} q_{3}+\omega_{1} q_{4}+\omega_{0} q_{3} \\
-\omega_{3} q_{1}+\omega_{1} q_{3}+\omega_{2} q_{4}+\omega_{0} q_{4} \\
\omega_{2} q_{1}-\omega_{1} q_{2}+\omega_{3} q_{4}-\omega_{0} q_{1} \\
-\omega_{1} q_{1}-\omega_{2} q_{2}-\omega_{3} q_{3}-\omega_{0} q_{2}
\end{array}\right], f_{s k}=\mathbf{f}_{\mathbf{q}, \mathbf{k}} \quad(\mathrm{k}=1,2)
\end{gathered}
$$

where $\omega_{0}$ is the orbital angular velocity, $\mathbf{q}=\left[q_{1}, q_{2}, q_{3}, q_{4}\right]^{T}$ and the measurements of the satellite angular velocity terms $\omega_{l}(l=1, \ldots, 3)$ are considered as independent measured inputs as illustrated in Section 5.1. The model of each of these two adaptive observers for $k=1,2$ is given by

$$
\left\{\begin{array}{l}
\dot{\overline{\bar{x}}}_{k}(t)=\bar{A} \hat{\bar{x}}_{k}(t)+\bar{W} f\left(\hat{\bar{x}}_{k}, y, t\right)+\bar{L}\left(\bar{y}_{k}-\hat{\bar{y}}_{k}\right)+\frac{1}{2} \hat{k} \bar{W} \bar{H}\left(\bar{y}_{k}-\hat{\bar{y}}_{k}\right)+\bar{D} \hat{f}_{s, k} \\
\hat{\bar{y}}_{k}=\hat{w}_{k}=\bar{C} \overline{\bar{x}}_{k}
\end{array}\right.
$$

In this case, the term $\hat{f}_{s, k}$ represents the estimated fault vector affecting the $k$-th attitude sensor.

Remark 7. The estimated fault vectors $\hat{f}_{s, 1}$ and $\hat{f}_{s, 2}$ are directly exploited as two sets of diagnostic signals $\xi_{i}(i=10, \ldots, 13)$ and $\xi_{i}(i=14, \ldots, 17)$, respectively, for the FDI task.

These estimates allow the isolation of the attitude sensor affected by a possible fault since only the NLGA adaptive observer specifically exploiting the faulty attitude sensor measurements provide a fault estimate $\hat{f}_{s, k}(k=1,2)$ different from zero with the assumption of a single fault occurring at any time.

However, it is worth noting that, actually, both of these NLGA adaptive observers result to be sensitive also to any angular velocity sensor fault $f_{\omega_{l}}(l=1, \ldots, 3)$. In fact, the angular velocity sensor faults $f_{\omega_{l}}$ directly affects, through the use of the corresponding measured sensor outputs as independent inputs, the observer dynamic model and then the residual signal $\bar{y}_{k}-\hat{\bar{y}}_{k}$ driving the adaptive fault estimate. Since the same angular velocity measurements are exploited as independent inputs by both of the adaptive observers, in case of any angular velocity sensor fault $f_{\omega_{l}}(l=$ $1, \ldots, 3)$, both of the provided fault estimates $\hat{f}_{s, k}(k=1,2)$ result to be different from zero and with the same behaviour.

Moreover, since the adaptive observers are specifically designed with respect to the occurrence of attitude sensor faults, the provided signals $\hat{f}_{s, k}(k=1,2)$ result to be accurate fault estimates only in case of attitude sensor faults $\mathbf{f}_{\mathbf{q}, \mathbf{k}}$, whilst they do not represent accurate estimates of the actual faults in case of any angular velocity sensor fault $f_{\omega_{l}}(l=1, \ldots, 3)$. On the other hand, considering the occurrence of possible satellite angular velocity sensor faults $f_{\omega_{l}}(l=1, \ldots, 3)$, starting from the nonlinear spacecraft model (13), the NLGA can be exploited in order to design an adaptive observer 
as described in Section 5.2. In particular, this adaptive observer provide accurate estimates of the angular velocity faults and results to be independent of the aerodynamic disturbance and actuator and sensor faults affecting the reaction wheel subsystems.

The state vector $\bar{x}$ of this NLGA adaptive observer is defined as follows:

$$
\bar{x}=\left[\begin{array}{c}
\left(q_{1}^{2}-q_{2}^{2}-q_{3}^{2}+q_{4}^{2}\right) \\
\left(-q_{1}^{2}+q_{2}^{2}-q_{3}^{2}+q_{4}^{2}\right) \\
\left(-q_{1}^{2}-q_{2}^{2}+q_{3}^{2}+q_{4}^{2}\right) \\
2\left(q_{1} q_{2}+q_{3} q_{4}\right) \\
2\left(q_{1} q_{3}+q_{2} q_{4}\right) \\
2\left(q_{1} q_{4}+q_{2} q_{3}\right) \\
2\left(q_{1} q_{2}-q_{3} q_{4}\right) \\
2\left(q_{1} q_{3}-q_{2} q_{4}\right) \\
2\left(q_{2} q_{3}-q_{1} q_{4}\right)
\end{array}\right]
$$

Therefore, considering the generic nonlinear model (25) and the related adaptive observer model (31), the following terms can be defined for this observer:

$$
\begin{aligned}
& \bar{A}=\left[\begin{array}{ccccccccc}
0 & 0 & 0 & 0 & 0 & 0 & 0 & \omega_{0} & 0 \\
0 & 0 & 0 & 0 & 0 & 0 & 0 & 0 & 0 \\
0 & 0 & 0 & 0 & -\omega_{0} & 0 & 0 & 0 & 0 \\
0 & 0 & 0 & 0 & 0 & 0 & 0 & 0 & 0 \\
0 & 0 & \omega_{0} & 0 & 0 & 0 & 0 & 0 & 0 \\
0 & 0 & 0 & 0 & 0 & 0 & -\omega_{0} & 0 & 0 \\
0 & 0 & 0 & 0 & 0 & \omega_{0} & 0 & 0 & 0 \\
-\omega_{0} & 0 & 0 & 0 & 0 & 0 & 0 & 0 & 0 \\
0 & 0 & 0 & 0 & 0 & 0 & 0 & 0 & 0
\end{array}\right], \bar{C}=I_{9}, \bar{D}=I_{9}, \bar{W}=I_{9} \\
& \left.\begin{array}{c}
f(\bar{x}, y, t)=\left[\begin{array}{c}
-2 \omega_{2}\left(q_{1} q_{3}+q_{2} q_{4}\right)+2 \omega_{3}\left(q_{1} q_{2}-q_{3} q_{4}\right) \\
2 \omega_{1}\left(q_{2} q_{3}-q_{1} q_{4}\right)-2 \omega_{3}\left(q_{1} q_{2}+q_{3} q_{4}\right) \\
-2 \omega_{1}\left(q_{1} q_{4}+q_{2} q_{3}\right)+2 \omega_{2}\left(q_{1} q_{3}-q_{2} q_{4}\right) \\
-2 \omega_{2}\left(q_{2} q_{3}-q_{1} q_{4}\right)+\omega_{3}\left(-q_{1}^{2}+q_{2}^{2}-q_{3}^{2}+q_{4}^{2}\right) \\
-2 \omega_{1}\left(q_{1} q_{2}-q_{3} q_{4}\right)+\omega_{2}\left(q_{1}^{2}-q_{2}^{2}-q_{3}^{2}+q_{4}^{2}\right) \\
\omega_{1}\left(-q_{1}^{2}-q_{2}^{2}+q_{3}^{2}+q_{4}^{2}\right)-2 \omega_{3}\left(q_{1} q_{3}-q_{2} q_{4}\right) \\
2 \omega_{1}\left(q_{1} q_{3}+q_{2} q_{4}\right)-\omega_{3}\left(q_{1}^{2}-q_{2}^{2}-q_{3}^{2}+q_{4}^{2}\right) \\
2 \omega_{3}\left(q_{1} q_{4}+q_{2} q_{3}\right)-\omega_{2}\left(-q_{1}^{2}-q_{2}^{2}+q_{3}^{2}+q_{4}^{2}\right) \\
2 \omega_{2}\left(q_{1} q_{2}+q_{3} q_{4}\right)-\omega_{1}\left(-q_{1}^{2}+q_{2}^{2}-q_{3}^{2}+q_{4}^{2}\right)
\end{array}\right] \\
-2 f_{\omega_{2}}\left(q_{1} q_{3}+q_{2} q_{4}\right)+2 f_{\omega_{3}}\left(q_{1} q_{2}-q_{3} q_{4}\right) \\
2 f_{\omega_{1}}\left(q_{2} q_{3}-q_{1} q_{4}\right)-2 f_{\omega_{3}}\left(q_{1} q_{2}+q_{3} q_{4}\right) \\
-2 f_{\omega_{1}}\left(q_{1} q_{4}+q_{2} q_{3}\right)+2 f_{\omega_{2}}\left(q_{1} q_{3}-q_{2} q_{4}\right) \\
-2 f_{\omega_{2}}\left(q_{2} q_{3}-q_{1} q_{4}\right)+f_{\omega_{3}}\left(-q_{1}^{2}+q_{2}^{2}-q_{3}^{2}+q_{4}^{2}\right) \\
-2 f_{\omega_{1}}\left(q_{1} q_{2}-q_{3} q_{4}\right)+f_{\omega_{2}}\left(q_{1}^{2}-q_{2}^{2}-q_{3}^{2}+q_{4}^{2}\right) \\
f_{\omega_{1}}\left(-q_{1}^{2}-q_{2}^{2}+q_{3}^{2}+q_{4}^{2}\right)-2 f_{\omega_{3}}\left(q_{1} q_{3}-q_{2} q_{4}\right) \\
2 f_{\omega_{1}}\left(q_{1} q_{3}+q_{2} q_{4}\right)-f_{\omega_{3}}\left(q_{1}^{2}-q_{2}^{2}-q_{3}^{2}+q_{4}^{2}\right) \\
2 f_{\omega_{3}}\left(q_{1} q_{4}+q_{2} q_{3}\right)-f_{\omega_{2}}\left(-q_{1}^{2}-q_{2}^{2}+q_{3}^{2}+q_{4}^{2}\right) \\
2 f_{\omega_{2}}\left(q_{1} q_{2}+q_{3} q_{4}\right)-f_{\omega_{1}}\left(-q_{1}^{2}+q_{2}^{2}-q_{3}^{2}+q_{4}^{2}\right)
\end{array}\right]
\end{aligned}
$$

where the elements of the additive fault vector $f_{a}$ that is actually estimated by the adaptive observer are functions of the observer state vector $\bar{x}$ and actual satellite angular velocity sensor faults $f_{\omega_{l}}$ $(l=1, \ldots, 3)$. In the same way, considering the satellite angular velocity terms $\omega_{l}(l=1, \ldots, 3)$ as independent measured inputs as illustrated in Section 5.1, the nonlinear terms of the adaptive observer are functions of the observer state vector $\bar{x}$ and satellite angular velocities $\omega_{l}(l=1, \ldots, 3)$. Therefore, the model of this adaptive observer is given by:

$$
\left\{\begin{array}{l}
\dot{\hat{x}}(t)=\bar{A} \hat{\bar{x}}(t)+\bar{W} f(\hat{\bar{x}}, y, t)+L(\bar{y}-\hat{\bar{y}})+\bar{D} \hat{f}_{a} \\
\hat{\bar{y}}=\bar{C} \hat{\bar{x}}
\end{array}\right.
$$


where the term $\hat{f}_{a}$ represents the estimated additive fault vector defined in (67).

Remark 8 . The estimated fault vector $\hat{f}_{a}$ is directly exploited as a set of diagnostic signals $\xi_{i}$ $(i=18, \ldots, 26)$ also for the FDI task.

The use of the NLGA allows to design an adaptive observer whose each state vector element is characterised by dynamics actually sensitive only to a specific couple of physical angular velocity sensor faults $f_{\omega_{l}}(l=1, \ldots, 3)$ through the mathematical relations of $f_{a, 1}$ in (67). Therefore, the provided diagnostic signals $\xi_{i}(i=18, \ldots, 26)$ can be organised as a generalised scheme.

In particular, the three diagnostic signals $\xi_{18}, \xi_{21}, \xi_{25}$ are sensitive only to the two angular velocity sensor faults $f_{\omega_{2}}, f_{\omega_{3}}$ and not sensitive to the fault $f_{\omega_{1}}$. The three diagnostic signals $\xi_{19}, \xi_{23}, \xi_{24}$ are sensitive only to the two angular velocity sensor faults $f_{\omega_{1}}, f_{\omega_{3}}$ and not sensitive to the fault $f_{\omega_{2}}$. Finally, the three diagnostic signals $\xi_{20}, \xi_{22}, \xi_{26}$ are sensitive only to the two angular velocity sensor faults $f_{\omega_{1}}, f_{\omega_{2}}$ and not sensitive to the fault $f_{\omega_{3}}$. These diagnostic signals allow for the accurate isolation of the angular velocity sensor actually affected by a possible fault through a proper crosscheck and decision logic.

Once a faulty angular velocity sensor has been correctly detected and isolated, the estimate $\hat{f}_{\omega_{l}}$ $(l=1, \ldots, 3)$ of the actual fault $f_{\omega_{l}}(l=1, \ldots, 3)$ affecting the sensor can be derived by means of the mathematical relations of $f_{a}$ in (67) and by exploiting the available attitude measurements thanks to the assumption of a single angular velocity sensor fault occurring at any time.

\section{DIAGNOSTIC SIGNAL CROSS-CHECK SCHEME}

The FDI task is achieved by means of a proper cross-check procedure of the diagnostic signals and exploiting a suitable decision logic to correctly detect and isolate the occurred fault, with the assumption of single fault at any time.

It is important to observe that in this paper the fault estimates $\xi_{1}, \ldots, \xi_{26}$ obtained by the designed NLGA adaptive filters are directly exploited as diagnostic signals also for the FDI task. These diagnostic signals are simultaneously checked to detect and isolate any of the considered actuator and sensor faults, without making any a-priori assumption regarding the occurring type of fault. Moreover, due to the presence of measurement noise, thresholds have to be properly selected for the generated diagnostic signals $\xi_{1}, \ldots, \xi_{26}$ in order to achieve the best performances in terms of false alarm and missed detection rates. The values reported in Table I have been empirically selected for each diagnostic signal by means of a $6-\sigma$ rule in a fault-free condition without parametric uncertainty.

Table I. Selected residual threshold values.

\begin{tabular}{cccccc}
\hline Diagnostic signal: & Threshold: & Diagnostic signal: & Threshold: & Diagnostic signal: & Threshold: \\
\hline$\xi_{1}$ & $2.58 \cdot 10^{-2}$ & $\xi_{10}$ & $3.67 \cdot 10^{-5}$ & $\xi_{18}$ & $5.54 \cdot 10^{-5}$ \\
$\xi_{2}$ & $3.06 \cdot 10^{-2}$ & $\xi_{11}$ & $3.65 \cdot 10^{-5}$ & $\xi_{19}$ & $2.85 \cdot 10^{-5}$ \\
$\xi_{3}$ & $3.00 \cdot 10^{-2}$ & $\xi_{12}$ & $4.08 \cdot 10^{-5}$ & $\xi_{20}$ & $5.11 \cdot 10^{-5}$ \\
$\xi_{4}$ & $2.52 \cdot 10^{-2}$ & $\xi_{13}$ & $1.45 \cdot 10^{-5}$ & $\xi_{21}$ & $8.28 \cdot 10^{-5}$ \\
$\xi_{5}$ & 0.5298 & $\xi_{14}$ & $3.67 \cdot 10^{-5}$ & $\xi_{22}$ & $6.95 \cdot 10^{-5}$ \\
$\xi_{6}$ & 0.5496 & $\xi_{15}$ & $3.65 \cdot 10^{-5}$ & $\xi_{23}$ & $8.72 \cdot 10^{-5}$ \\
$\xi_{7}$ & 0.4074 & $\xi_{16}$ & $4.08 \cdot 10^{-5}$ & $\xi_{24}$ & $7.71 \cdot 10^{-5}$ \\
$\xi_{8}$ & 0.7128 & $\xi_{17}$ & $1.45 \cdot 10^{-5}$ & $\xi_{25}$ & $6.61 \cdot 10^{-5}$ \\
$\xi_{9}$ & $1.52 \cdot 10^{-2}$ & & & $\xi_{26}$ & $8.11 \cdot 10^{-5}$
\end{tabular}

\subsection{Fault Detection and Isolation Scheme for Actuator and Flywheel Spin Rate Sensor Faults}

Assuming a single fault at any time, possible faults affecting the actuated reaction wheel motor torques or flywheel spin rate measurements can be detected and isolated by cross-checking the nine signals $\xi_{1}, \ldots, \xi_{9}$ provided by the NLGA adaptive observers relying on the variables (55), (58) and (62) described in Section 5.4, as follows: 
1. Firstly, the two sets diagnostic signals $\xi_{1}, \ldots, \xi_{4}$ and $\xi_{5}, \ldots, \xi_{8}$ are analyzed. The first four signals represent accurate estimates only of possible faults affecting the actuated control torques, whilst the other four represent accurate estimates only of possible flywheel spin rate sensor faults. Since each of the corresponding observers is sensitive only to the couple of possible actuator and sensor faults affecting a specific reaction wheel, the faulty reaction wheel subsystem can be easily detected and isolated as a fault estimate exceeds a properly selected threshold.

2. Subsequently, a check on the additional diagnostic signal $\xi_{9}$ allows to precisely isolate also the type of the occurred fault (i.e. its location in the reaction wheel subsystem) since this signal is sensitive only to a combination of sensor faults and insensitive to any actuator fault. The diagnostic signal $f_{\xi_{9}}$ does not exceed the selected threshold after the occurrence of any actuator fault, whilst it does after the occurrence of any flywheel spin rate sensor fault.

\subsection{Fault Detection and Isolation Scheme for Satellite Attitude and Angular Velocity Sensor Faults}

The occurrence of a possible fault affecting the measurements of the satellite attitude and angular velocity sensors can be detected and isolated by cross-checking the two sets of four diagnostic signals $\xi_{10}, \ldots, \xi_{13}$ and $\xi_{14}, \ldots, \xi_{17}$ and the nine diagnostic signals $\xi_{18}, \ldots, \xi_{26}$ provided by the NLGA adaptive observer relying on the variables (63) and (66) described in Section 5.5, respectively, as follows:

1. Firstly, the two sets $\xi_{10}, \ldots, \xi_{13}$ and $\xi_{14}, \ldots, \xi_{17}$ are compared. Since each set exploits the measurements of a different attitude sensor and of the same angular velocity sensors, the two sets show different signal behaviours in case of attitude sensor faults and the same behaviours in case of angular velocity sensor faults. Hence, it can be recognized if an attitude or angular velocity sensor fault has occurred.

2. A faulty attitude sensor is isolated by checking which is the only set with signals exceeding the selected thresholds.

3. On the other hand, a faulty angular velocity sensor is isolated by checking the three signals of the set $\xi_{18}, \ldots, \xi_{26}$ not sensitive to each specific angular velocity sensor fault and thus not exceeding the selected thresholds.

\section{SIMULATION RESULTS}

Some results achieved in Matlab/Simulink ${ }^{\circledR}$ are reported in the following sections in order to show the effectiveness of the proposed diagnosis scheme.

\subsection{Simulation Parameters and Fault Scenarios}

The satellite body is modelled as a rectangular parallelepiped whose the principal dimensions are $d=0.6 \mathrm{~m}, w=2 \mathrm{~m}$, and $h=7.5 \mathrm{~m}$ (depth $\mathbf{x}$ width $\mathbf{x}$ height), $\mathbf{r}_{\mathbf{c p}}=[0.10,0.15,-0.35] \mathrm{m}$ is the aerodynamic torque displacement vector, while the inertia values are $I_{x x}=330 \mathrm{~kg} \cdot \mathrm{m}^{2}$, $I_{y y}=280 \mathrm{~kg} \cdot \mathrm{m}^{2}, I_{z z}=60 \mathrm{~kg} \cdot \mathrm{m}^{2}$.

A circular orbit at an altitude of $350 \mathrm{~km}$ and null inclination, with a low Earth equatorial orbit radius $R=6728.140 \mathrm{~km}$ is considered. The atmosphere density is $\rho=\rho_{\max }=6 \cdot 10^{-11} \mathrm{~kg} / \mathrm{m}^{3}$, the drag coefficient is $C_{D}=2.2$, the orbital velocity is $V=8187.63 \mathrm{~m} / \mathrm{s}$, and the Earth's gravitational constant $\mu=39.86004418 \cdot 10^{13} \mathrm{~m}^{3} / \mathrm{s}^{2}$.

The reaction wheels maximal torque is set to $0.75 \mathrm{Nm}$. The viscous and Coulomb friction coefficients are $b=5.16 \cdot 10^{-6} \mathrm{Nms}$ and $c=0.8795 \cdot 10^{-3} \mathrm{Nm}$, respectively. A flywheel moment of inertia $I_{r w}=0.05 \mathrm{~kg} \cdot \mathrm{m}^{2}$ and initial flywheel spin rate values $\omega_{0}=$ $[1500,1500,-1500,-1500]^{T} \mathrm{rpm}$ for the four considered reaction wheels are assumed.

A standard Sliding Mode Controller (SMC) has been implemented in the ACS. A gradual attitude change manoeuvre is considered, commencing at $t_{\text {man }}=10 \mathrm{~s}$ from the initial attitude $\mathbf{q}_{0}=$ $[-0.0570,0.3180,0.1663,0.9316]^{T}$ to reach the final one $\mathbf{q}=[-0.0429,0.2732,0.1815,0.9437]^{T}$. 
These quaternion vectors correspond to $\left[\phi_{0}, \theta_{0}, \psi_{0},\right]^{T}=[-15,35,25]^{T}$ deg and $[\phi, \theta, \psi,]^{T}=$ $[-12,30,25]^{T}$ deg for the attitude in Euler angles (i.e. roll, pitch and yaw angles), respectively. Assuming a single fault at any time, four additive fault scenarios commencing at $t_{\text {fault }}=20 \mathrm{~s}$ are considered:

1. Actuator fault: $f_{M_{2}}=-a_{M} \omega_{r w_{2}}-b_{M}$ with $a_{M}$ linearly passing from zero at $t=20 \mathrm{~s}$ to $0.003 \mathrm{Nms}$ at $t=30 \mathrm{~s}$ and $b_{M}=0.05 \mathrm{Nm}$;

2. Flywheel sensor fault: $f_{\omega_{r w, 2}}=-a_{\omega_{r w}}$ with $a_{\omega_{r w}}$ linearly passing from zero at $t=20 \mathrm{~s}$ to $-0.5235 \mathrm{rad} / \mathrm{s}=-100 \mathrm{rpm}$ at $t=45 \mathrm{~s}$ and then changing from $-0.5235 \mathrm{rad} / \mathrm{s}=-100 \mathrm{rpm}$ at $t=50 \mathrm{~s}$ to $-0.2618 \mathrm{rad} / \mathrm{s}=-50 \mathrm{rpm}$ at $t=55 \mathrm{~s}$;

3. Attitude sensor fault: $f_{\mathbf{q}, \mathbf{1}}$ additive on the first quaternion measurement, corresponding to a constant bias of $8.7266 \cdot 10^{-4} \mathrm{rad}=0.05 \mathrm{deg}$ on the roll angle measurements;

4. Angular velocity sensor fault: $f_{\omega_{3}}=-a_{\omega} \sin \left(b_{\omega} t\right)$ with $a_{\omega}$ linearly passing from zero at $t=20 \mathrm{~s}$ to $6.9808 \cdot 10^{-4} \mathrm{rad} / \mathrm{s}$ at $t=40 \mathrm{~s}$ and $b_{\omega}=0.05 \pi \mathrm{rad} / \mathrm{s}=0.025 \mathrm{~Hz}$.

It is noted that the proposed diagnosis scheme does not assume any a-priori hypothesis regarding the fault type and that the diagnosis system takes into account the possible occurrence of faults affecting any actuator or sensor of the satellite ADCS. Moreover, the generic additive faults can be used to represent different fault causes (e.g. mechanical, electrical, thermal, magnetic damages and malfunctions, parameter variations, etc.), and behaviours (e.g. lock-in-place, failure, loss of effectiveness, drift, bias, etc.). Sensor noises are modelled by Gaussian processes with zero mean. Standard deviations equal to $3 \mathrm{arcsec}, 3 \mathrm{arcsec} / \mathrm{s}$ and $1 \mathrm{rpm}$ are assumed for the attitude measured in Euler angles, satellite angular speed and flywheel spin rate measurements, respectively. A simulation time of $60 \mathrm{~s}$ with a sampling time of $0.025 \mathrm{~s}$ is considered.

\subsection{Fault Scenario 1 - Diagnosis of Actuator Faults}

In case of the actuator fault $f_{M_{2}}$, the FDI task can be carried out by cross-checking the five diagnostic signals $\xi_{1}, \ldots, \xi_{4}$ and $\xi_{9}$ described in Section (5.4), on the basis of the decision logic described in Section (6.1). Fig. 1 shows the four diagnostic signals $\xi_{1}, \ldots, \xi_{4}$, provided by the NLGA adaptive observers based on the state variables (55), and the diagnostic signal $\xi_{9}$, which is sensitive only to sensor faults and decoupled from the aerodynamic disturbance and actuator faults. In particular, the signal $\xi_{2}$ is sensitive to the couple of faults $f_{M_{2}}, f_{\omega_{r w, 2}}$, although it actually represents the correct estimate of $f_{M_{2}}$ only. The selected thresholds are depicted for each signal by means of red lines. It is possible to detect and isolate the faulty subsystem just by means of the four diagnostic signals $\xi_{1}, \ldots, \xi_{4}$. After the isolation of the faulty reaction wheel subsystem, a check on

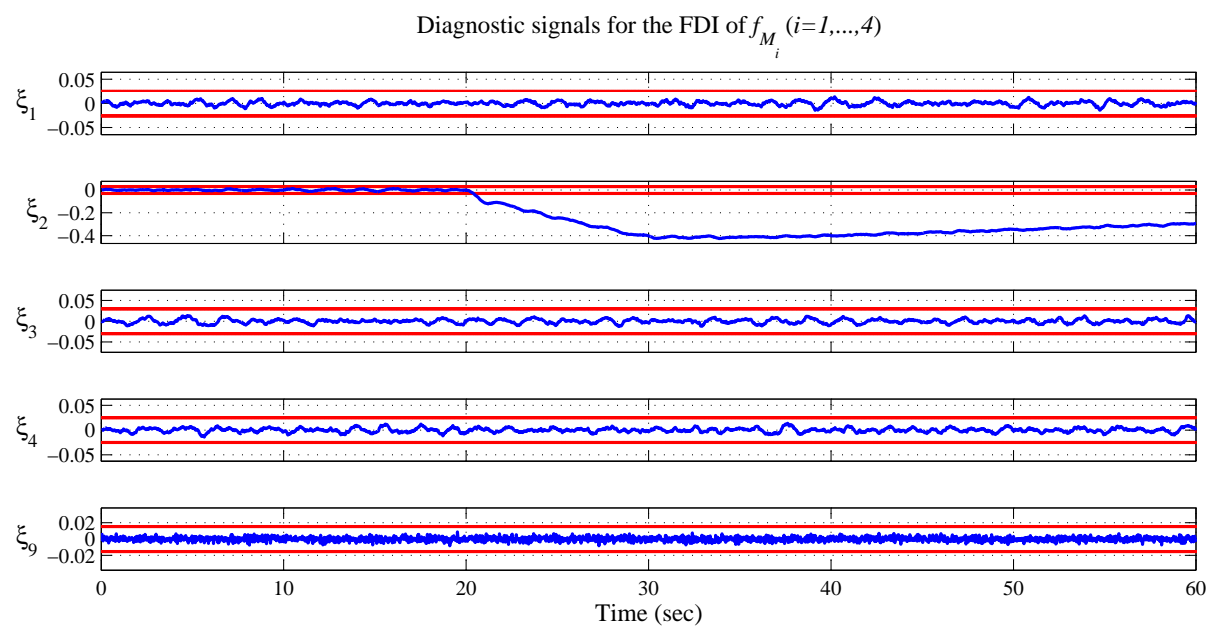

Figure 1. Actuator fault: four diagnostic signals sensitive to faults on a specific reaction wheel and additional residual signal sensitive only to sensor faults. 


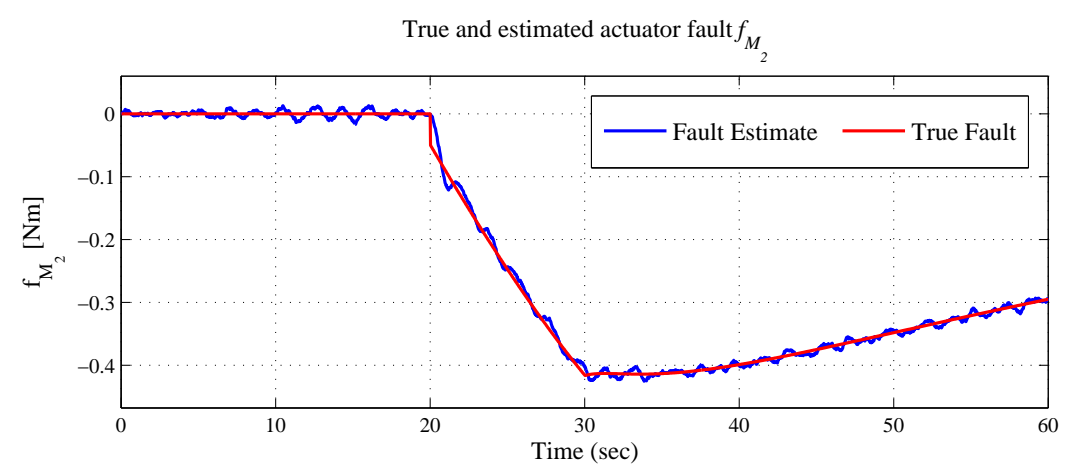

Figure 2. Estimate of the actuator fault $f_{M_{2}}$.

the signal $\xi_{9}$ allows to precisely isolate also the type of the occurred fault (i.e. its location) since this residual is sensitive only to sensor faults and insensitive to actuator faults. It does not exceed the selected threshold in case of actuator faults.

Once an actuator fault $f_{M_{i}}(i=1, \ldots, 4)$ has been detected and isolated, the corresponding estimate is directly given by the related diagnostic signal $\hat{f}_{M_{i}}=\xi_{i}(i=1, \ldots, 4)$, which has been exploited also for the FDI task. Fig. 2 shows the estimate $\hat{f}_{M_{2}}$ of the actuator fault $f_{M_{2}}$ provided by the corresponding NLGA adaptive observer. It can be seen that the adaptive observer provides an accurate estimate of the occurred fault, even in case of a generic fault function.

\subsection{Fault Scenario 2 - Diagnosis of Flywheel Spin Rate Sensor Faults}

On the other hand, in case of the flywheel spin rate sensor fault $f_{\omega_{r w, 2}}$, the FDI task can be carried out by cross-checking the five diagnostic signals $\xi_{5}, \ldots, \xi_{9}$ described in Sections (5.4), on the basis of the decision logic described in Section (6.1). Fig. 3 shows the four diagnostic signals $\xi_{5}, \ldots, \xi_{8}$ provided by the NLGA adaptive observers based on the variables (58). In particular, the signal $\xi_{6}$ is sensitive to the couple of faults $f_{M_{2}}, f_{\omega_{r w, 2}}$, although it actually represents the correct estimate of $f_{\omega_{r w, 2}}$ only. As already described, the diagnostic signal $\xi_{9}$ is sensitive only to flywheel sensor faults and decoupled from the aerodynamic disturbance and actuator faults and allows to precisely isolate also the type (i.e. its location) of the occurred fault since this residual is sensitive only to sensor faults and insensitive to actuator faults. In this case, it does exceed the selected threshold after the sensor fault occurrence. Hence, the occurred flywheel sensor fault can be correctly isolated thanks to the different behaviour of this additional diagnostic signal. Once a flywheel spin rate sensor fault $f_{\omega_{r w, j}}(j=1, \ldots, 4)$ has been detected and isolated, the corresponding estimate is directly given by the related diagnostic signal $\hat{f}_{\omega_{r w, j}}=\xi_{i}(i=6, \ldots, 9)$, which has been exploited also for the FDI task. Fig. 4 shows the estimate $\hat{f}_{\omega_{r w, 2}}$ of the sensor fault $f_{\omega_{r w, 2}}$.

Remark 9. It is worth noting that, even if there are detection delays to exceed thresholds, the fault estimates do not suffer from delay since these signals coincide with the diagnostic signals exploited also by the FDI system, which are available in real time, and there are no estimation filters to be activated and initialized after the fault isolation.

\subsection{Fault Scenario 3 - Diagnosis of Satellite Attitude Sensor Faults}

The occurrence of faults affecting one of the two considered attitude sensors can be detected and isolated by exploiting the first two NLGA adaptive observers described in Section 5.5.

The diagnostic signals $\xi_{10}, \ldots, \xi_{13}$ and $\xi_{14}, \ldots, \xi_{17}$ obtained by means of the corresponding two adaptive observers represent the correct estimates of the components of possible attitude sensor faults $\mathbf{f}_{\mathbf{q}, \mathbf{1}}$ and $\mathbf{f}_{\mathbf{q}, \mathbf{2}}$ affecting the first and second attitude sensor, respectively. Therefore, assuming a single fault occurring at any time, in case of attitude sensor fault the two observers, each providing the estimate of a possible additive fault $f_{\mathbf{q}, \mathbf{k}}(k=1,2)$ affecting a specific attitude sensor, are 


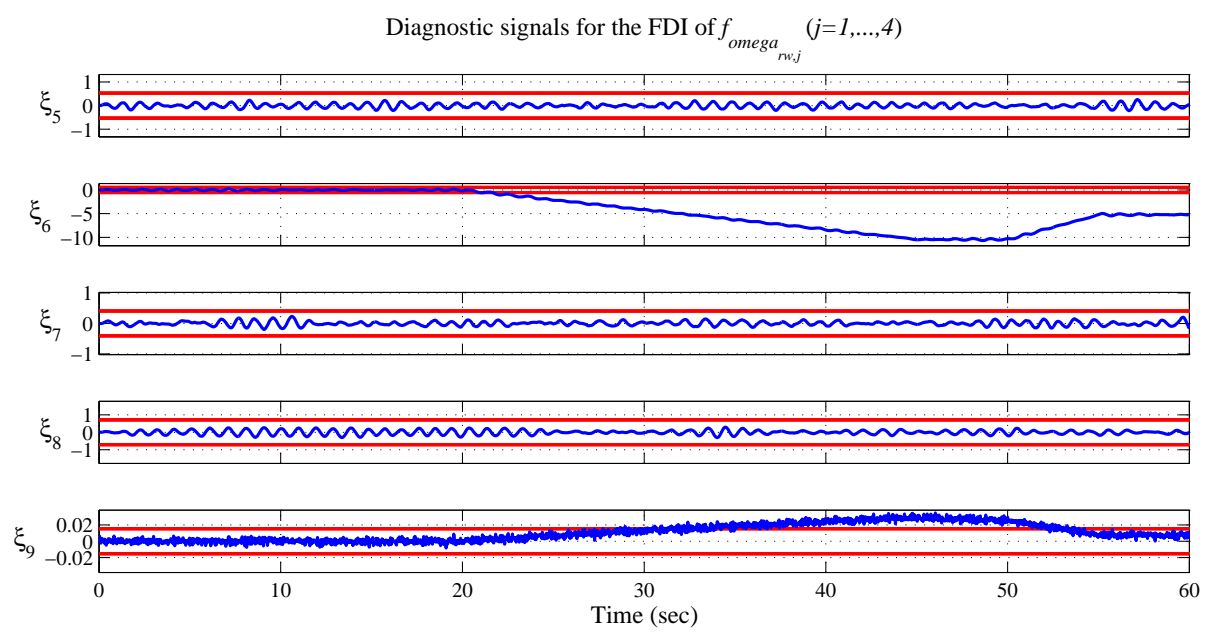

Figure 3. Flywheel sensor fault: four diagnostic signals sensitive to faults on a specific reaction wheel and additional residual signal sensitive only to sensor faults.

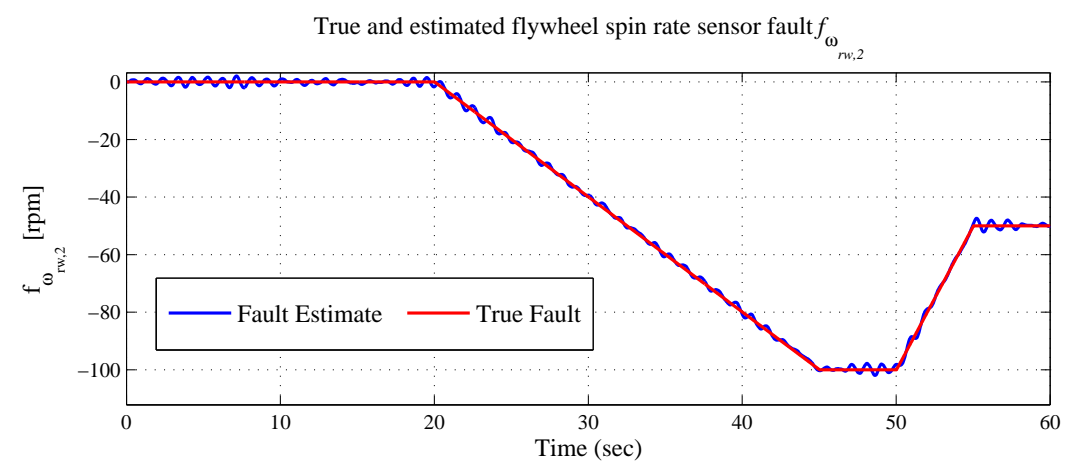

Figure 4. Estimate of the flywheel sensor fault $f_{\omega_{r w, 2}}$.

characterised by diagnostic signals with different behaviours, as described in Section 6.2. Fig. 5 shows on the left the diagnostic signals $\xi_{10}, \ldots, \xi_{13}$ provided by the adaptive observer exploiting the measurements of the first attitude sensor and angular velocity sensors the diagnostic signals $\xi_{14}, \ldots, \xi_{17}$ provided by the adaptive observer exploiting the measurements of the second attitude sensor and same angular velocity sensors. In can be seen that the two sets of diagnostic signals are characterised by different behaviours. Hence, the occurrence of a fault affecting a specific attitude sensor, which is feeding the set whose diagnostic signals exceed the selected thresholds, can be isolated as described in Section 6.2.

Fig. 6 shows the estimate $\hat{f}_{\mathbf{q}, \mathbf{1}}$ obtained once the considered additive fault $f_{\mathbf{q}, \mathbf{1}}$ affecting the first attitude sensor has been properly isolated. The estimates of the additive fault components are directly given by the related diagnostic signals $\hat{f}_{\mathbf{q}, 1}=\left[\xi_{10}, \xi_{11}, \xi_{12}, \xi_{13}\right]^{T}$, which have been exploited also for the FDI task.

\subsection{Fault Scenario 4 - Diagnosis of Satellite Angular Velocity Sensor Faults}

The occurrence of faults affecting any satellite angular velocity component can be detected and isolated by exploiting the three NLGA adaptive observers described in Section 5.5.

The diagnostic signals $\xi_{10}, \ldots, \xi_{13}$ and $\xi_{14}, \ldots, \xi_{17}$ are actually sensitive also to the occurrence of faults affecting any satellite angular velocity component since the two observers exploit also the angular velocity measurements as independent inputs. Therefore, assuming a single fault occurring at any time, in case of angular velocity sensor fault $f_{\omega_{3}}$ the two observers are characterised by 

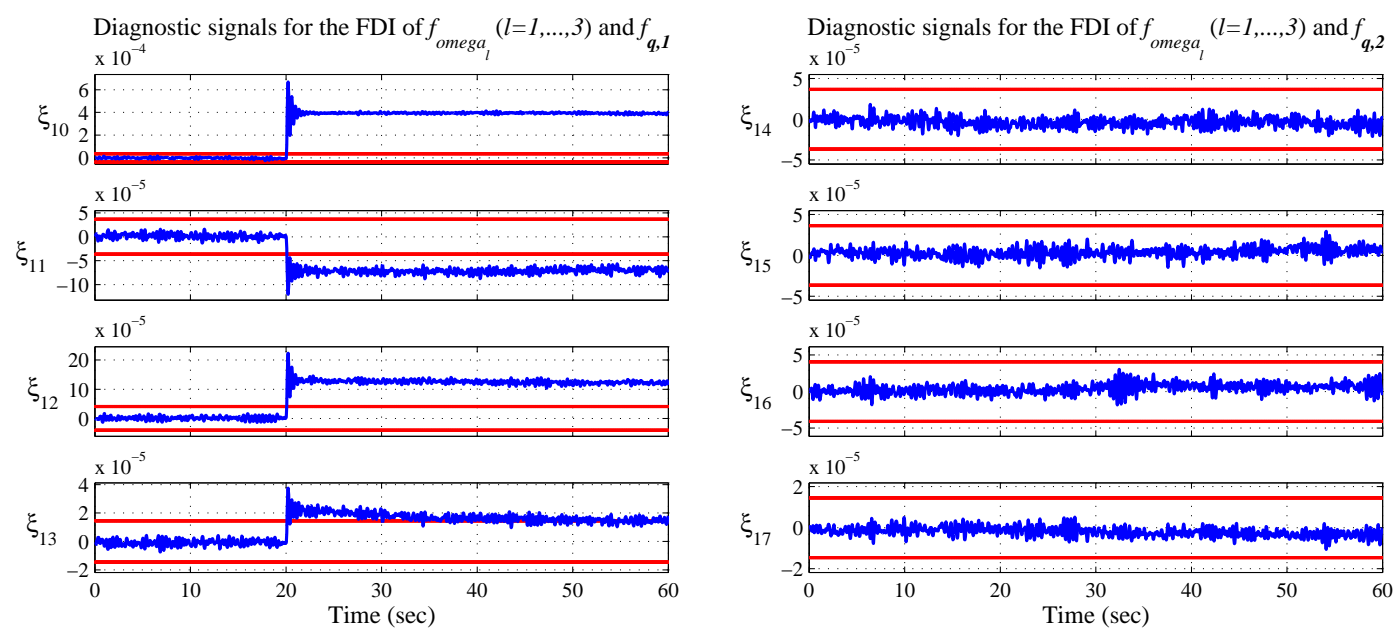

Figure 5. Attitude sensor fault: two sets of diagnostic signals provided by the adaptive observer exploiting the measurements of the first (left) and second (right) attitude sensor, respectively.
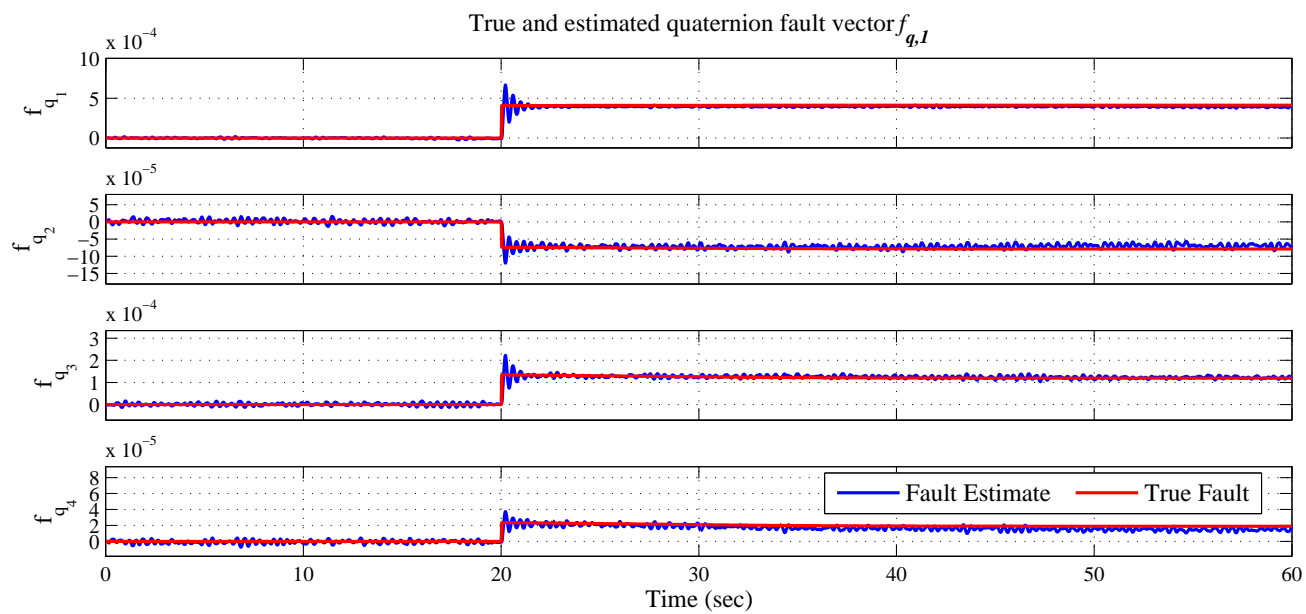

Figure 6. Estimate of the additive quaternion fault vector $f_{\mathbf{q}, \mathbf{1}}$ associated to the physical attitude sensor fault.

diagnostic signals with the same behaviours and exceeding the selected thresholds, since they exploit the same angular velocity measurements, as described in Section 6.2. However, it is not yet possible to isolate the specific faulty angular velocity sensor on the basis of these diagnostic signals only.

Therefore, once a fault affecting a generic angular velocity sensor has been detected, the nine diagnostic signals $\xi_{18}, \ldots, \xi_{26}$ have to be exploited in order to accurately isolate the specific faulty angular velocity sensor. In particular, Fig. 7 shows the three diagnostic signals $\xi_{18}, \xi_{21}, \xi_{25}$ that are sensitive only to the two angular velocity sensor faults $f_{\omega_{2}}, f_{\omega_{3}}$ and not sensitive to the fault $f_{\omega_{1}}$. Fig. 8 shows the three diagnostic signals $\xi_{19}, \xi_{23}, \xi_{24}$ that are sensitive only to the two angular velocity sensor faults $f_{\omega_{1}}, f_{\omega_{3}}$ and not sensitive to the fault $f_{\omega_{2}}$. Finally, Fig. 9 shows the three diagnostic signals $\xi_{20}, \xi_{22}, \xi_{26}$ that are sensitive only to the two angular velocity sensor faults $f_{\omega_{1}}$, $f_{\omega_{2}}$ and not sensitive to the fault $f_{\omega_{3}}$. In this case, in Fig. 9 the last three signals $\xi_{20}, \xi_{22}, \xi_{26}$, which results to be decoupled from possible faults affecting the third angular velocity sensor, do not exceed the selected thresholds. On the contrary, the other six signals generally result to be sensitive to the occurred angular velocity sensor fault.

Finally, Fig. 10 shows the estimate $\hat{f}_{\omega_{3}}$ obtained once the fault $f_{\omega_{3}}$ has been properly isolated. The estimate of the actual fault is mathematically derived directly from the diagnostic signals $\xi_{18}, \ldots, \xi_{26}$, which have been exploited to complete the fault isolation task. 

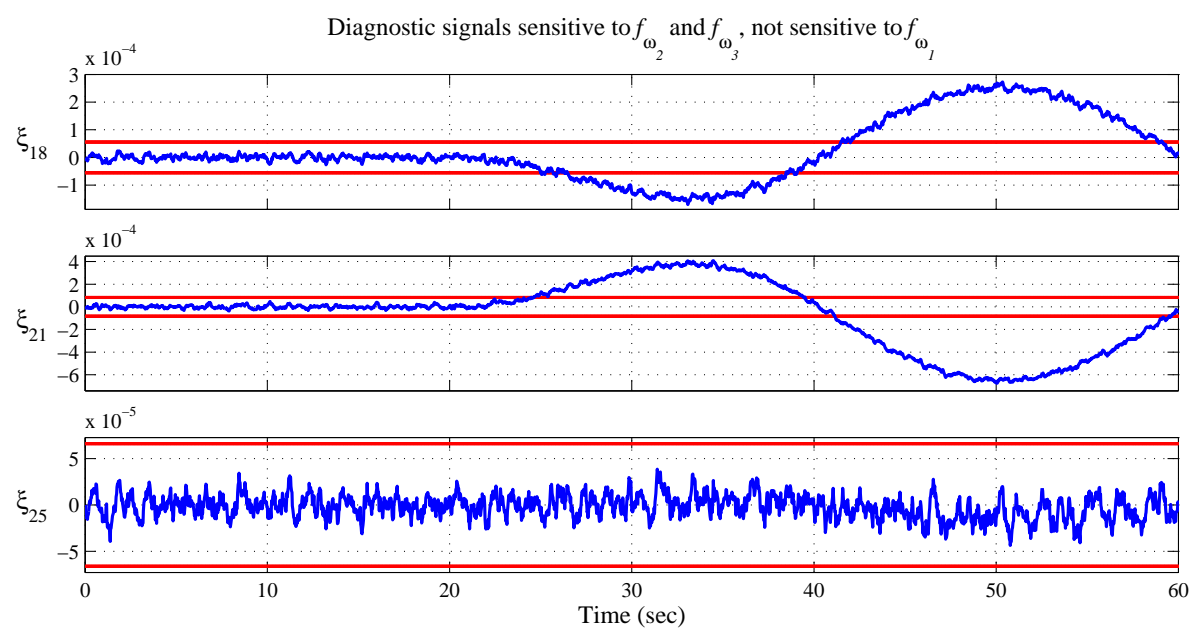

Figure 7. Angular velocity sensor fault: set of three diagnostic signals sensitive to $f_{\omega_{2}}$ and $f_{\omega_{3}}$, not sensitive to $f_{\omega_{1}}$.

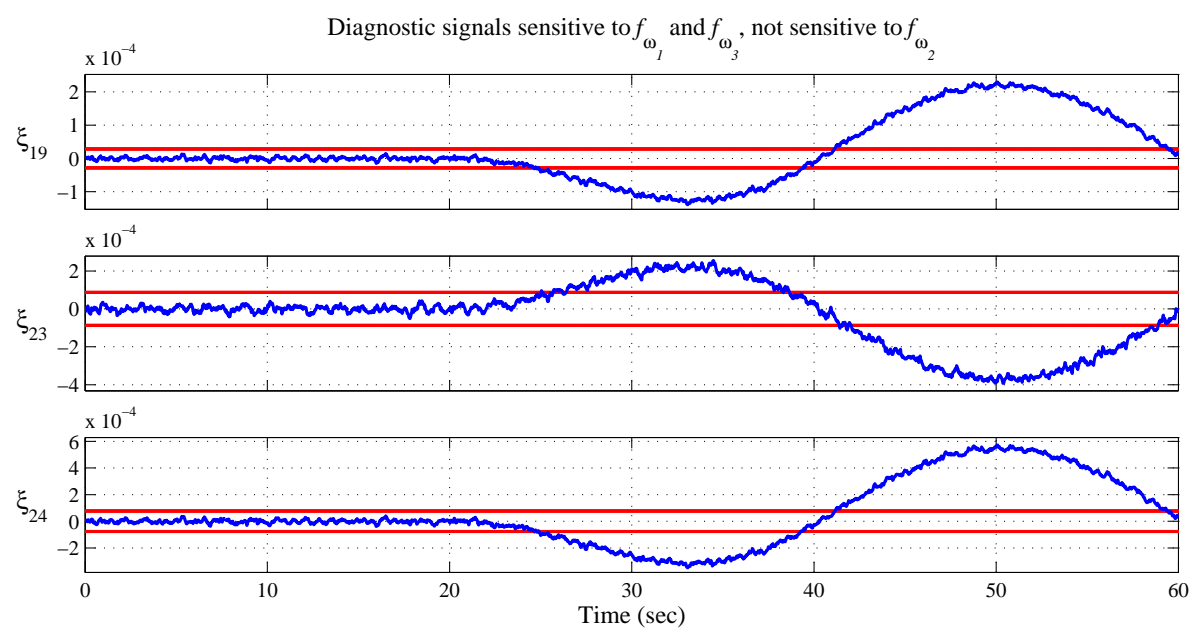

Figure 8. Angular velocity sensor fault: set of three diagnostic signals sensitive to $f_{\omega_{1}}$ and $f_{\omega_{3}}$, not sensitive to $f_{\omega_{2}}$.

\subsection{Robustness analysis}

In order to assess the robustness and reliability of the proposed fault detection and isolation scheme, several Monte-Carlo simulations have been performed in case of different parametric uncertainties. The following parameters have been assumed as uncertain in the actual satellite model or in the filters model with the following distributions and characteristic values directly reported in the following tables:

1. Drag coefficient $C_{D}$ in filter model: normal distribution with standard deviation $\sigma_{C_{D}}$ and mean (nominal) value $\mu_{C d}=2.2$;

2. Air density $\rho$ in satellite model: uniform distribution between $\rho_{\min }=2 \cdot 10^{-12} \mathrm{~kg} / \mathrm{m}^{3}$ and $\rho_{\text {max }}=6 \cdot 10^{-11} \mathrm{~kg} / \mathrm{m}^{3}$;

3. Earth gravitational constant $\mu$ in satellite model: uniform distribution between $\mu_{\min / \max }=$ $39.86004418 \pm 0.00000008 \cdot 10^{13} \mathrm{~m}^{3} / \mathrm{s}^{2}$;

4. Inertia vector $\mathbf{I}_{\text {sat }}$ in filter model: normal distributions with standard deviation $\sigma_{\text {Ist }}$ for each single component and mean (nominal) values $\mathbf{I}_{\mathbf{s a t}}=[\mathbf{3 3 0}, \mathbf{2 8 0}, \mathbf{6 0}] \mathrm{kg} \cdot \mathrm{m}^{\mathbf{2}}$; 


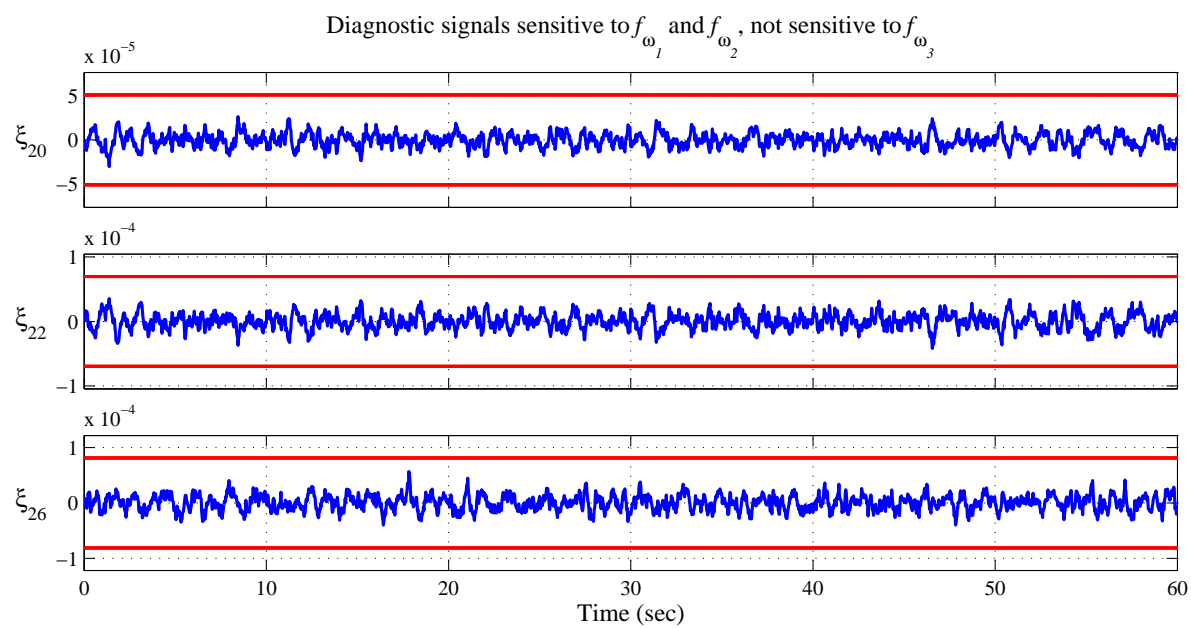

Figure 9. Angular velocity sensor fault: set of three diagnostic signals sensitive to $f_{\omega_{1}}$ and $f_{\omega_{2}}$, not sensitive to $f_{\omega_{3}}$.

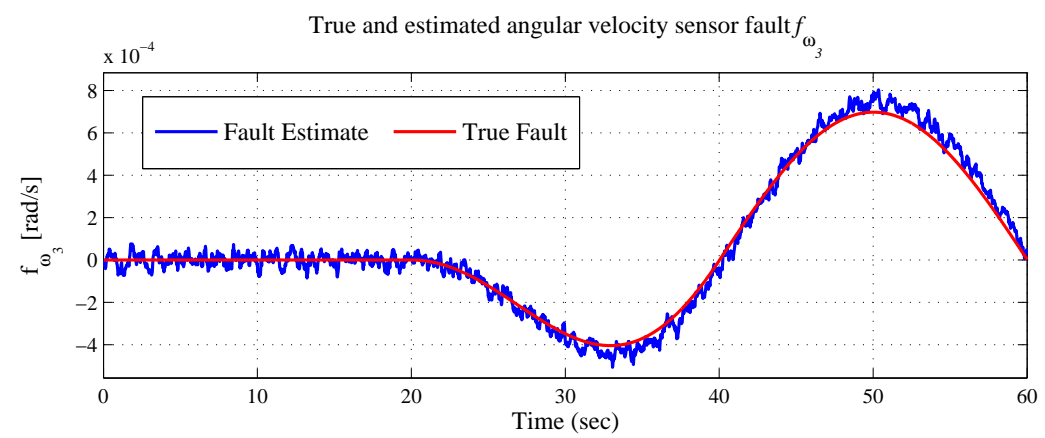

Figure 10. Estimate of the angular velocity sensor fault $f_{\omega_{3}}$.

5. Aerodynamic displacement vector $\mathbf{r}_{\mathbf{c p}}$ in filter model: normal distributions with standard deviation $\sigma_{r}$ for each single component and mean (nominal) value $\mathbf{r}_{\mathbf{c p}}=$ $[0.10,0.15,-0.35] \mathrm{m}$;

Remark 10. No uncertainties affecting the flywheel moments of inertia or reaction wheel friction parameters have been considered in this analysis, since the flywheel moments can be easily and accurately verified by means of laboratory tests during the satellite design phase, whilst any possible variation or uncertainty of the friction parameters with respect to the nominal values has been considered as actually due to mechanical actuator faults to be detected, isolated and estimated (e.g. due to loss of lubrication, mechanical bearing damages, etc.). Moreover, the accurate knowledge of the flywheel moment of inertia in the reaction wheel model allows to obtain accurate estimates of the actuator and flywheel spin rate sensor faults exploiting the proposed adaptive observers.

For performance analysis of the proposed fault detection and isolation scheme, Sensitivity (Sens), Specificity (Spec), and Accuracy (Accu) performance metrics are calculated as follows [26]:

$$
\text { Sens }=\frac{T P}{(T P+F N)} \quad \text { Spec }=\frac{T N}{(T P+F N)} \quad A c c u=\frac{T N+T P}{(T N+T P+F N+F P)}
$$

where true positive $(T P)$ represents the number of trials during which the fault is correctly detected and isolated over the whole number of trials of each Monte-Carlo trials run (correct detection), true negative $(T N)$ represents the number of trials which are correctly recognized as normal operating conditions, false positive $(F P)$ denotes the number of trials during which actual faulty operating conditions are incorrectly recognized as normal ones (missed detection or incorrect isolation), and 
false negative $(F N)$ represents to the number of actual normal conditions which are incorrectly recognized as faulty ones (false alarm). In particular, the accuracy performance index is equal to 1.00 in the best situation (100\% of correct fault isolations or rejections) and lower in case of some missed isolations or false alarms.

Each table reports the FDI performance results regarding a specific actual fault condition, i.e. actuator fault $f_{M_{2}}$ (Tables II and III), sensor fault $f_{\omega_{r w, 2}}$ (Table IV) or fault-free condition (Table V). Different standard deviation values have been implemented for the normal distributions of the parameters. Moreover, the number of trials for each test configuration and the numbers of TP, TN, FP and FN are reported along with the sensitivity, specificity and accuracy parameters.

For each performance index and counter, two different values are given in each table and uncertainty condition: the first one regards the number of TP, TN, FP or FN and the resulting Sens, Spec and Accu obtained taking into exam the diagnostic signals for the detection and isolation of a hypothetical fault $f_{M_{2}}$, the second one regards the signals taken into exam for the detection and isolation of a hypothetical fault $f_{\omega_{r w, 2}}$.

Remark 11. The robustness analysis has been performed only for actuator faults $f_{M_{i}}(\mathrm{i}=1, \ldots, 4)$ and flywheel spin rate sensor faults $f_{\omega_{r w}, j}(\mathrm{j}=1, \ldots, 4)$. In fact, it is worth noting that the models of the adaptive observers exploited for the FDD of satellite angular velocity and attitude sensor faults $f_{\mathbf{q}, \mathbf{k}}$ $(\mathrm{k}=1,2)$ and $f_{\omega_{l}}(\mathrm{l}=1, \ldots, 3)$ are actually functions only of the state variables and no other uncertain or time-varying parameters are involved. Therefore, these adaptive observers and the diagnosis of the related faults are automatically robust to any parameter uncertainty.

As it can be seen from the Tables II-V, both in stationary attitude and manouvre conditions, the proposed detection and isolation scheme results to be robust to several parameter uncertainties also in case of quite high uncertainty standard deviations. In particular, since the aerodynamic disturbance is analytically decoupled in the observer and filter models, the corresponding diagnostic signals result to be completely independent from any uncertainty affecting the drag coefficient and air density values. Moreover, the effect of the uncertainty affecting the gravitational constant, with the considered bound limits based on realistic values related to the Earth gravitational field, results to be actually negligible. The considered realistic and very narrow experimental bounds of the gravitational parameter confirm the assumption of a gravitational disturbance model almost perfectly known.

Finally, the proposed scheme results to be generally robust also to uncertainties affecting the knowledge of the satellite moments of inertia and aerodynamic displacement vector $\mathbf{r}_{\mathbf{c p}}$. Just in case of actual actuator fault and in the manouvre condition, it can be seen that some missed detections and isolations or false alarms can occur due to the presence uncertainty affecting the knowledge of the satellite moments of inertia, mainly due to the modulation and amplification of the model error related to the parametric uncertainty by the satellite angular velocity values associated to the manoeuvre. In this situation, the uncertainty leads to a misleading recognition of the occurred fault type on the basis of the obtained additional diagnostic signal $\xi_{9}$ given by the additional NLGA residual filter.

Therefore, the accurate knowledge of the satellite moments of inertia results to be the most critical aspect regarding the robustness and reliability of the proposed fault detection and isolation scheme in case of actual actuator faults. However, it can be seen that the obtained isolation error consists essentially in the wrong classification of the actual type of an occurring actual fault, due to the structure of the exploited decision logic scheme. In the absence of any actuator or sensor fault, or in case of flywheel spin rate sensor faults, the proposed scheme allows to obtain the correct fault rejection or isolation even in case of manouvre and inertial parameter uncertainties.

\section{CONCLUSION}

This paper presented a novel practical scheme for diagnosis of actuator and sensor faults that affect the attitude determination and control system (ADCS) of a low Earth orbit satellite. A structural analysis was exploited to qualitatively assess the detectability and isolability of defects in the ADCS. This analysis showed that partial hardware sensor redundancy is required in order 
Table II. Robustness analysis for the FDI of hypothetical faults $f_{M_{2}}, f_{\omega_{r w, 2}}$ in case of actual fault $f_{M_{2}}$ w.r.t. parametric uncertainties (percent standard deviations w.r.t. nominal values) without attitude change manouvre.

\begin{tabular}{|c|c|c|c|c|c|c|c|c|c|c|c|c|c|c|c|}
\hline$f_{M_{2}}$ & $\mid$ Trials $\mid$ & $T P_{M 2}$ & $T N_{M 2}$ & $F P_{M 2}$ & $F N_{M 2}$ & $\operatorname{Sens}_{M 2}$ & $\operatorname{Spec}_{M 2}$ & $A c c u_{M 2}$ & $\mid T P_{\omega 2}$ & $T N_{\omega 2}$ & $F P_{\omega 2}$ & $F N_{\omega 2} \mid$ & $\operatorname{Sens}_{\omega 2}$ & $S_{p e c}{ }_{\omega 2}$ & $A c c u_{\omega 2}$ \\
\hline$\rho$ & 100 & 100 & 0 & 0 & 0 & 1.00 & - & 1.00 & 0 & 100 & 0 & 0 & - & 1.00 & 1.00 \\
\hline$C_{D}: \sigma=2 \%$ & 100 & 100 & 0 & 0 & 0 & 1.00 & - & 1.00 & 0 & 100 & 0 & 0 & - & 1.00 & 1.00 \\
\hline$C_{D}: \sigma=10 \%$ & 100 & 100 & 0 & 0 & 0 & 1.00 & - & 1.00 & 0 & 100 & 0 & 0 & - & 1.00 & 1.00 \\
\hline $\mathbf{r}_{\mathbf{c p}}: \sigma=\mathbf{3} \%$ & 100 & 100 & 0 & 0 & 0 & 1.00 & - & 1.00 & 0 & 100 & 0 & 0 & - & 1.00 & 1.00 \\
\hline $\mathbf{r}_{\mathbf{c p}}: \sigma=\mathbf{5} \%$ & 100 & 100 & 0 & 0 & 0 & 1.00 & - & 1.00 & 0 & 100 & 0 & 0 & - & 1.00 & 1.00 \\
\hline $\mathbf{I}_{\text {sat }}: \sigma=\mathbf{5} \%$ & 100 & 100 & 0 & 0 & 0 & 1.00 & - & 1.00 & 0 & 100 & 0 & 0 & - & 1.00 & 1.00 \\
\hline
\end{tabular}

Table III. Robustness analysis for the FDI of hypothetical faults $f_{M_{2}}, f_{\omega_{r w, 2}}$ in case of actual fault $f_{M_{2}}$ w.r.t. parametric uncertainties (percent standard deviations w.r.t. nominal values) with attitude change manouvre.

\begin{tabular}{|c|c|c|c|c|c|c|c|c|c|c|c|c|c|c|c|}
\hline$f_{M_{2}}$ & Tria & $T P_{M}$ & $T N_{N}$ & ${ }_{2} F P_{\Lambda}$ & $F N_{M 2}$ & Sens & Spec & $A c c$ & $T P_{\omega 2}$ & $T N_{\omega 2}$ & $F P_{\omega 2}$ & $F N_{\omega 2}$ & $\operatorname{Sens}_{\omega 2}$ & $S_{\text {pec }} 2$ & $A c c u_{\omega 2}$ \\
\hline & $\begin{array}{ll}0 & 100\end{array}$ & 100 & 0 & 0 & 0 & 1.00 & - & 1.00 & 0 & 100 & 0 & 0 & - & 1.00 & 1.00 \\
\hline I $_{\text {sat }}: \sigma=\mathbf{2} \%$ & 100 & 98 & 0 & 2 & 0 & 1.00 & 0.00 & 0.98 & 0 & 98 & 0 & 2 & 0.00 & 1.00 & 0.98 \\
\hline $\mathbf{I}_{\text {sat }}: \sigma=\mathbf{5} \%$ & 100 & 56 & 0 & 44 & 0 & 1.00 & 0.00 & 0.56 & 0 & 56 & 0 & 44 & 0.00 & 1.00 & 0.56 \\
\hline
\end{tabular}

Table IV. Robustness analysis for the FDI of hypothetical faults $f_{M_{2}}, f_{\omega_{r w, 2}}$ in case of actual fault $f_{\omega_{r w, 2}}$ w.r.t. parametric uncertainties, both with and without attitude change manouvre.

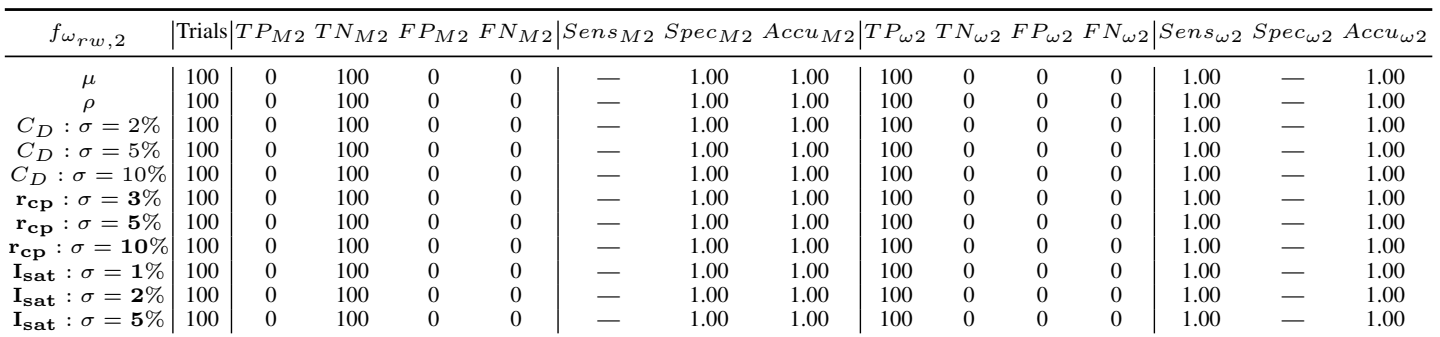

Table V. Robustness analysis for the FDI of hypothetical faults $f_{M_{2}}, f_{\omega_{r w, 2}}$ in case of actual fault-free condition w.r.t. parametric uncertainties, both with and without attitude change manouvre.

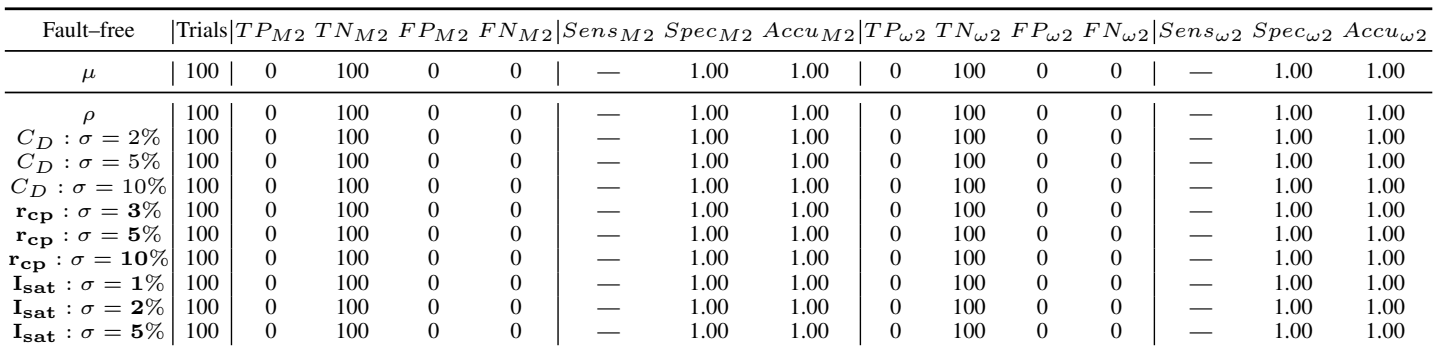

to achieve complete isolation of all possible sensor faults. The proposed diagnosis algorithm exploited fault estimates provided by adaptive observers as diagnostic signals, and a generalised estimation filter design was obtained using an approach based on an augmented spacecraft. Aerodynamic disturbance decoupling was achieved using the nonlinear geometric approach. The use of adaptive fault estimation filters allowed to estimate generic fault functions without needing any a-priori information about fault internal models. Simulation results served to assess the effectiveness of the proposed diagnosis scheme and verified that the method is able to achieve quick and correct fault detection and isolation and also provide accurate fault estimates. A robustness and reliability analysis with respect to system parameter uncertainties and disturbances verified very satisfactory performances of the proposed fault diagnosis scheme. Further developments could concern the integration of the proposed scheme in an active fault-tolerant control system. 


\section{REFERENCES}

1. Blanke, M., Kinnaert, M., Lunze, J., and Staroswiecki, M. (2016). Diagnosis and Fault-tolerant Control, 3rd Edition. 3rd edition, Springer-Verlag Berlin Heidelberg.

2. Isermann, R. (2011). Fault Diagnosis Applications: Model-based Condition Monitoring: Actuators, Drives, Machinery, Plants, Sensors, and Fault-tolerant Systems. Springer-Verlag Berlin Heidelberg.

3. Ding, S.X. (2013). Model-based Fault Diagnosis Techniques: Design Schemes, Algorithms, and Tools. 2nd edition, Springer-Verlag London.

4. Chen, J., and Patton, R.J. (1999). Robust Model-based Fault Diagnosis for Dynamic Systems. Kluwer Academic Publishers.

5. Bokor, J. and Szabó, Z. (2009). Fault detection and isolation in nonlinear systems. Annual Reviews in Control, 33, $113-123$.

6. Edelmayer, A., Bokor, J., Szabó, Z. and Szigeti, F. (2004). Input reconstruction by means of system inversion: A geometric approach to fault detection and isolation in nonlinear systems. International Journal of Applied Mathematics and Computer Science, 14(2), 189-199.

7. De Persis, C., and Isidori, A. (2001). A geometric approach to nonlinear fault detection and isolation. IEEE Transactions on Automatic Control, 45, 853-865.

8. Patton, R., Uppal, F., Simani, S., and Polle, B. (2008). Reliable fault diagnosis scheme for a spacecraft attitude control system. Proceedings of the Institution of Mechanical Engineers, Part O: Journal of Risk and Reliability, $222,139-152$

9. Wu, Q., and Saif, M. (2009). Model-based robust fault diagnosis for satellite control systems using learning and sliding mode approaches. Journal of Computers, 4(10), 1022-1032.

10. Azarnoush, H. (2010). Fault diagnosis in spacecraft attitude control system: a model-based approach. LAP LAMBERT Academic Publishing.

11. Baldi, P., Blanke, M., Castaldi, P., Mimmo, N., and Simani, S. (2015). Combined Geometric and Neural Network Approach to Generic Fault Diagnosis in Satellite Reaction Wheels. 9th IFAC Symposium on Fault Detection, Supervision and Safety for Technical Processes - SAFEPROCESS'15, Paris (France), 48(21), 194-199.

12. Mattone, R., and De Luca, A. (2006). Nonlinear fault detection and isolation in a three-tank heating system. IEEE Transactions on Control Systems Technology, 14(6), 1158-1166.

13. Baldi, P., Blanke, M., Castaldi, P., Mimmo, N., and Simani, S. (2016). Combined Geometric and Neural Network Approach to Generic Fault Diagnosis in Satellite Actuators and Sensors. 20th IFAC Symposium on Automatic Control in Aerospace - ACA 2016, Sherbrooke (Canada), 49(17), 432-437.

14. Chen, W., and Saif, M. (2007). Observer-based fault diagnosis of satellite systems subject to time-varying thruster faults. Journal of Dynamic Systems, Measurement and Control, Transactions of the ASME, 129(3), 352-356.

15. Meskin, K., and Khorasani, N. (2007). Fault detection and isolation in a redundant reaction wheels configuration of a satellite. Proceedings of the IEEE International Conference on Systems, Man and Cybernetics - ICSMC 2007, Montréal (Canada), CD-Rom, 3153-3158.

16. Hajiyev, C. (2009). Innovation Approach Based Sensor FDI in LEO Satellite Attitude Determination and Control System. Kalman Filter: Recent Advances and Applications. I-Tech Education and Publishing KG, Vienna, Austria.

17. Zhang, J., Swain, A.K., and Nguang, S.K. (2016). Robust Observer-based Fault Diagnosis for Nonlinear Systems Using Matlab ${ }^{\circledR}$, Springer International Publishing AG Switzerland.

18. Jiang, B., Staroswiescki, M., and Cocquempot, V. (2006). Fault Accommodation for Nonlinear Dynamic Systems. IEEE Transactions on Automatic Control, 51(9), 1578-1583.

19. Tan, C.P., and Edwards, C. (2001). Reconstruction of Sensor Faults Using a Secondary Sliding Mode Observer. Proceedings of the 40th IEEE Conference on Decision and Control, 1, 579-584.

20. Izadi-Zamanabadi, R., Larsen, J.A. (2007). A Fault tolerant Control Supervisory System development Procedure for Small Satellites: The AAUSAT-II case. 17th IFAC Symposium on Automatic Control in Aerospace, 40(7), 289-294.

21. Lorentzen, M. Blanke, and H. Niemann (2003). Structural analysis - A case study of the romer satellite. 5th IFAC Symposium on Fault Detection, Supervision and Safety for Technical Processes - SAFEPROCESS'03, 36(5), 185190.

22. Wie, B. (2008). Space Vehicle Dynamics and Control (2nd ed.). AIAA Education Series.

23. Sidi, M.J. (1997). Spacecraft dynamics and control. Cambridge University Press, USA.

24. Carrara, V., da Silva, A.G., and Kuga, H.K. (2012). A dynamic friction model for reaction wheels. 1st IAA Conference on Dynamic and Control of Space Systems, 145, 343-352.

25. Edwards, C., Lombaerts, T. and Smaili, H. (2010). Fault Tolerant Flight Control: A Benchmark Challenge. SpringerVerlag Berlin Heidelberg, Germany.

26. Nozari, H. A., Simani, S., Shoorehdeli, M. A. (2012). Model-based robust fault detection and isolation of an industrial gas turbine prototype using soft computing techniques. Neurocomputing, 91, 29-47. 